\title{
Studien über die Pathogenese des Glaucoms.
}

\author{
Von \\ Dr. Rich. Ulrich, \\ Privatdoeent an der Universität Strassburg.
}

Hierzu Tafel IX. X (13 Figuren).

I. Irisfiltration.

Wer sich heut zu Tage mit der Pathogenese des Glaucoms befassen will, kann die Frage der Irisfiltration nicht ausser Acht lassen. Denn wenn es ja auch klar ist, dass wir in ihr keineswegs das Fundament des glaucomatösen Processes zu sehen haben, so ist sie doch immerhin ein Factor, der in dem Krankheitsverlauf und namentlich in der Therapie eine bedeutende Rolle spielt. Die Frage ist dann anch in den letzten Jahren mehrfach in Angriff genommen worden, doch lässt sich die kleine Litteratur noch recht gut übersehen und ich würde es kaum für nöthig halten, an dieser Stelle den gegenwärtigen Stand der Discussion wiederzugeben, wenn nicht die in dem "Lehrbuch der Anatomie der Sinnesorgane von G. Schwalbe enthaltene Kritik*) mir eine Erwiderung zur Pflicht machte. Diese Erwiderung soll indess

*) p. 179 u. 214. 
nur in einer Zusammenstellung dessen, was bis jetzt in dieser Richtung publicirt wurde, bestehen, das Facit wird sich dann von selbst ergeben.

Im Jahre 1875 veröffentlichte Knies*) Versuche, die er an Kaninchen behufs Erforschung intraocularer FlüssigkeitsStrömung angestellt hatte. Knies hatte Injectionen von Forro-Cyan-Kalium in den Glaskörper des lebenden Thieres gemacht, nach einigen Stunden das Thier getödtet und die Vertheilung des Ferro-Cyan-Kaliums im Auge durch Eisenchlorid fixirt. Er entdeckte dabei die bekannte blaue Linie, die am Sclero-Cornealrand in die Cornea einsetzt, sich in der Sclera nach hinten wendet und dann im Orbitalgewebe endet. Eine befriedigende Erklärung für dies Phänomen wusste Knies nicht recht zu geben; dagegen wurde das Faktum sehr bald von $\mathrm{L}$. Weiss**) bestätigt.

Im Jahre 1880 wiederholte ich ***) die Knies'schen intraocularen Injectionen von Ferro-Cyan-Kalium und wies nach, dass die blaue Linie in der Corneo-Sclera nur die Fortsetzung sei von einem blauen Streifen, der die Irisperipherie quer durchsetze und vordere und hintere Kammer mit einander verbinde. Ich nannte ihn Filtrationsstreifen der Iris. Um mich ron dem physiologischen Charakter desselben zu überzeugen, wandte ich mich später der schonenderen Methode der Subeutan-Injectionen von Ferro-Cyan-Kalium zu und es glückte mir mittelst derselben nicht nur die Ergebnisse der directen Injection bestatigt zu finden, sondern auch zu zeigen, dass nach Paracentese der Cornea insofern ein anderes Resultat eintrete, als die stärkste Blaufärbung am Pupillarrand der Tris und der entsprechenden Stelle der Hornhaut zu finden sei. Ieh schloss aus diesen Versuchen, dass am intacten Auge der Humor aqueus in die Vorderkammer in der Gegend der Kammerbucht eintrete, indem durch die Iriswurzel hindurch eine Filtration stattfinde, dass aber nach Paracentese der Cornea das regenerirte Kammerwasser am hyperämischen Pupillarrand erscheine, indem es von den Gefässschlingen desselben secernirt werde.

*) Virchow's Archiv Bd. 65.

**) Graefe's Archiv XXV, 2.

***) Graefe's Archiv XXVI. 3. 
Auf dem internationalen Aerzte-Congress in London hatte dann Weber*) in Darmstadt ron subcutanen Injectionen von Ferro-Cyan-Kalium an Kaninchen berichtet, die aber in Bezug auf die Frage der Irisfiltration negativ ausfielen. Das negative Resultat lag meiner Ansicht nach an der Versuchs-Modification, die zur Anwendung kam.

Wenn man auf irgend eine Weise ein Salz in die Blutbahn eines Thieres einbringt, so ist es ja selbstrerständlich, dass man nach einem gewissen Zeitraum dies Salz in allen blutführenden Geweben wiederfindet, also anch in dem flüssigkeitsreichen Irisgewebe. Will man nun die Frage prüfen, ob dieses Salz, ausser aus den Irisgefässen auch aus der hintern Augenkammer in die Irisperipherie eindringt, so hommt alles darauf an, dass man den Versuch zur richtigen, experimentell auszuprobirenden, Zeit unterbricht und das Resultat rasch fixirt. Denn es ist ja klar, dass, wenn man zu lange wartet, sowohl von den Irisgefässen aus als auch von der Irisperipherie her, wo die Flüssigkeit der hintern Kammer eindringt, eine diffuse Verbreitung des Salzes in den communicirenden Gewebsmaschen der gesammten Regenbogenhaut eintreten und eventuell bei Fixation durch Eisenchlorid zu einer diffusen Blaufärbung führen wird.

Weber verfuhr nun so, dass er 7 Stunden lang jede Stunde 2 Pravaz'sche Spritzen voll Ferro Cyan-Kalium-Lösung subcutan injicirte und dann das Thier tödtete: das Resultat war in Bezug auf die Iris ein vollständig negatives, wie zu erwarten war.

Während die bisherigen Beobachtungen am getödteten Thier und an enucleirten Augen angestellt waren, wurde im Jahre 1882 durch Ehrlich **) ein Mittel in die ExperimentalPhysiologie eingeführt, welches ermöglichte, das Auftreten des Kammerwassers in der vorderen Kammer an lebenden Thieren zu studiren. Ehrlich verwandte, wie bekannt, das Fluorescin zur subcutanen Injection. Das Resultat, zu dem er kam, lässt sich kurz dahin zusammenfassen, dass bei normalen KaninchenAugen der Humor aqueus in der Peripherie die vordere Kam-

*) Transactions of the international med. Congress, London, Ophthalmology.

**) Ueber provocirte Fluorescenz-Erscheinungen am Auge. Deutsche medic. Wochensehrift No.2 ff. 
mer betrete, dagegen nach Paracentese der Cornea am Pupillarrand.

In demselben Jahre bemerkt Pflüg er*), dass er bei Wiederholung der subcutanen Injectionen von Fluorescin „die Ergebnisse der Ehrlich'schen Experimente vollständig bestätigt gefunden habe, sowohl die fluorescirende linie in der vordern Kammer, als das leuchtende Hypopyon."

Pflüger injicirte aber auch Fluorescin direct in den Glaskörper, aspirirte 7 Stunden nachher Humor aqueus und fand ihn frei von Fluorescin. Als er ferner mittelst Manometer-Versuchs Fluorescin in die vordere Kammer einbrachte, färbte sich die Iris; 6 Stunden nachher punktirte er den Glaskörper, derselbe zeigte deutliche Fluorescenz. „Aus diesen Versuchen möchte ich nicht schliessen, dass der Flüssigkeitsstrom von der vordern Kammer normaliter durch die Zonula nach hinten in den Glaskörper geht, soviel aber, dass der Humor aqueus nicht von dem Corpus vitreum geliefert wird."

Während also Pflüger die Ehrlich'schen nach subcutaner Injectionsmethode gewonnenen Resultate bestätigt, sind ihm, wie es scheint, 2 Einzelversuche directer Bulbus-Injection missglückt: bei dem ersten erhielt er gar kein Resultat und bei dem zweiten ein solches, dessen Verlässlichkeit er selbst bezweifelt.

Widerspruch gegen die Beobachtungen Ehrlich's erhob sich indess im folgenden Jahre von Seiten ${ }^{* *}$ ) SchölerUhth of f's.

Subcutane sowie directe Glaskörper-Injectionen ron Fluorescin führten diese Autoren zu der Ansicht, dass sowohl im intacten Kaninchenauge als auch nach Punction der Cornea das Kammerwasser am Pupillarrand in der Vorderkammer erscheine.

Bei diesem auffallenden Gegensatze in den Resultaten, welche trotz Anwendung derselben Methode Ehrlich und Pflüger einerseits und Schöler-Uhthoff andererseits erzielten, sah ich ***) mich veranlasst, die Fluorescin-Injectionen gleichfalls zu wiederholen.

*) Zehenders Monatsblätter 1882, p. 81 .

**) Jahresbericht der Klinik aus dem Jahre 1882.

***) Archiv fiir Augenheilkunde, Beitrag zu den Untersuchungen etc. p. 153. 
Ich wällte zu den Versuchen Albino-Kaninchen, bei denen die helle Iris leichte Färbungen besser erkennen lässt und wandte ausserdem mit Erfolg eine Combination der FerroCyan-Kalium- und Fluorescin-Methode an, indem ich beide Stoffe zu gleichen Theilen mischte und die gelöste Mischung subcutan injizirte.

Diese Combination erlaubte zunächst, am lebenden Thier die Fluorescin-Färbung, sodann am getödteten anatomisch die Berliner-Blaufärbung zu beobachten und ihre Identität mit der ersteren za constatiren. Indem ich ferner noch die Beobachtung der Fluorescenz im Spectrallicht hinzufügte, fand ich meine im Jahre 1880 aufgestellte Ansicht bestätigt, dass am intacten Auge das Kammerwasser in der Peripherie, am punctirten Auge am Pupillarrand die Vorderkammer betrete.

Dies Resultat fand eine weitere Bestätigung, wie Herr Prof. Schmidt - Rimpler in der Heidelberger OphthalmologenVersammlung 1884 mittheilte, durch Versuche, die mittelst Fluorescin in der Marburger Augenklinik angestellt wurden and deren Veroffentlichung in einer Inaugural-Dissertation berorsteht.

Die bisher berichteten Injectionen haben das Gemeinsame, dass sie mittelst diffusibler Stoffe angestellt wurden. Indessen ans verschiedenen Gründen war es mir schon im Jahre 1880 wünschenswerth ersehienen, in der Injection körniger Farbstoffe eine Controle $z u$ besitzen und ich hatte schon damals Injectionen in den Glaskörper mit Verreibung von chinesischer Tusche in $3 / 4$ pCt. Salzwasser versucht. Die damaligen Experimente missgluckten insofern, als sich jedesmal eine so heftige entzündliche Reaction einstellte, dass ich die erhaltenen Resultate für physiologische Strömungs-Verhältnisse nicht als massgebend ansehen konnte und auf die Verfolgung derselben verzichtete.

Vor Kurzem hat nun Baumgarten*) beiläufig über Glaskörper-Injectionen von Zinnober und Anilinblau be-

*) v. Graefes Archiv XXIX. 3. 
richtet und angegeben, er habe niemals Theilchen den Stoffe in der Cornea oder dem Canalis Schlemmii getroffen, auffallende entzündliche Reactionen erwähnt er nicht.

Ich wurde dadurch in der Ueberzeugung bestärkt, dass meine früheren Misserfolge mit Tusche-Injectionen auf zufällige Nebenumstände, eine ron aussen stammende Infection, zurückzuführen seien, und beschloss deshalb, unter Ergreifung besonderer Vorsichtsmaassregeln, dieselben zu wiederholen. In der That wurde in der zweiten Versuchsreihe jene erste entzündliche Reaction vermieden. Es erfolgte in den Tagen nach der Injection kaum eine schwache pericorneale Rothung, dabei liess sich die Tusche mit unbewaffnetem Auge oder mittelst des Ophthalmoskops leicht im Glaskörper constatiren.

Das Resultat der Versuche, die ich gewohnlich nach 24, resp. 48 Stunden durch Todtung des Thieres abbrach, war ein doppeltes. - Bei einer Anzahl von Augen fand sich bei der anatomischen Untersuchung der ganze vordere Abschnitt des Bulbus, also der vorderste Theil des Corpus vitreum, der perilentale Raum, die Iriswurzel und die Kammerbucht vollständig frei von Tusche: es hatte keinerlei Locomotion der Injectionsmasse in dieser Richtung stattgefunden. Dahingegen liess sich schon makroskopisch eine Anhäufung von Tusche auf der Papille, der Excavation entsprechend, constatiren und ferner zeigte der Enucleations-Querschnitt des Nervus opticus den Centralcanal durch einen schwarzen Tuschepunkt markirt. Die mikroskopische Untersuchung bestätigte, dass sich der Farbstoff von der Excavation aus längs der Gefässe in den Centralcanal begeben hatte und mit den Gefässen den Sehnerv auch wieder verliess.

Die Tusche-Partikelchen waren im Allgemeinen im Innern von Lymphzellen eingeschlossen, so dass man den Eindruck bekam, dass diese die Locomotion vermittelt 
hätten. In das Zellgewebe der Orbita hinein habe ich die Färbung nicht weiter verfolgt.

Wenn diese Versuche nun auch bei der Frage der Irisfiltration nicht mitsprechen konnten - die Injectionsmasse war ja gar nicht nach vorne gelangt - so wich doch das Resultat von dem früher bei Injection von FerroCyan-Kaliumlösung in den Glaskorper erhaltenen so erheblich $a b$, dass ich mich nach einer Erklärung umsehen musste.

A priori war es ja klar, dass die Locomotion körniger Farbstoffe im Glaskörper schwerer von statten gehen würde, als die von Ferro-Cyan-Kaliumlosung oder Fluoreszin und folgerichtig musste ich auf ein Mittel sinnen, den intraocularen Flüssigkeitsstrom zu verstärken. Nun hatte ich früher bei subcutanen Injectionen von Fluorescin die Erfahrung gemacht, dass die Existenz einer vorderen Synechie, eines Lencoma adhärens bei Kaninchen augenscheinlich eine beträchtliche Beschleunigung der Kammerwasser-Secretion zur Folge hatte. Auf den derartig operirten Augen erschien, auch wenn die Heilung seit Jahr und Tag beendet war, die Fluorescenz stets früher und reichlicher in der Vorderkammer als bei dem gesunden Partner. Diese Erfahrung machte ich mir zu Nutze, vollführte bei einem alten Kaninchen, bei dem eine vordere Synechie nach oben seit ca. einem halben Jahr auf dem einen Auge bestand, auf diesem sowie auf dem gesunden anderen Auge eine Tusche-Injection und tödete das Thier nach 44 Stunden. Die anatomische Untersuchung ergab auf dem gesunden Auge ein negatives Resultat. Auf dem Auge mit vorderer Synechie war die Reaction auf den Eingriff während des Lebens etwas stärker als gewöhnlich: es trat eine Iritis ein, übrigens mit auffallend geringer äusserer Gefäss-Injection. - Anatomisch zeigte es sich, dass die in den Glaskörper injicirte Tusche eine Locomotion nach vorne erfahren hatte, in vollständig analoger 
Weise, wie ich es früher von den Ferro-Cyan-KaliumVersuchen beschrieben habe.

Es fand sich rings um den Linsenäquator eine An häufung von Farbstoff, eine Infiltration der Processus ciliares und der Iriswurzel mit demselben an der dem Linsenäquator entsprechenden Stelle und eine beträchtliche Fullung der Fontana'schen Räume; dagegen war im Uebrigen die hintere Flache der Iris bis zum Pupillarrand frei von Tusche. Die beigegebenen Abbildungen (Fig. 1-3) dispensiren mich von weiterer Beschreibung. Kein Unbefangener kann hiernach zweifeln, dass die Tusche-Partikelchen die Iriswurzel durchsetzt baben, um so in die Kammerbucht zu gelangen. Bemerkenswerth ist es, dass die Farbstofftheilchen nicht in Lymphzellen enthalten waren, sondern frei im Gewebe lagen.

Zieht man nun das Facit der bis jetzt bekannt gewordenen Versuchsresultate, so ergibt sich aus obiger Zusammenstellung Folgendes: 1) Alle Experimentatoren sind darin einig, dass nach Paracentese der Cornea das regenerirte Kammerwasser am Pupillarrand die Vorderkammer betritt. (Centraler Eintritt des Kammerwassers.) 2) Mit Ausnahme ron Scholer-Uhthoffs Versuchen ergeben alle anderen, dass bei intactem Auge der Humor aqueus die Vorderkammer in der Peripherie betritt. (Peripherer Eintritt des Kammerwassers.) 3) Eine Anzahl von Injectionen in den Glaskörper verliefen ohne Resultat, indem eine Fortbewegung der Injectionsmasse nach vorne nicht erfolgte.

Die Erfahrung, dass auf die direkten Injectionen ein gewisser Prozentsatz negativer Erfolge entfällt, wird wohl keinem der angeführten Forscher erspart geblieben sein. Im Einzelfall lässt sich selten entscheiden, woran die Schuld liegt; dagegen ist im Allgemeinen ein solches Misslingen wohl $\mathrm{zu}$ verstehen. Selbst wenn man mit feinster Stich-Canule arbeitet, sieht man in der Mehrzahl der Fälle dem Ausziehen derselben einen Austritt von 
Glaskörper unter die Conjunctiva nachfolgen und es resultirt eine bleibende Fistel, durch die der intraoculare Druck beeinflusst werden muss. Nun ist es aber a priori höchst wahrscheinlich und wird durch die Injections-Versuche noch bekraftigt, dass die Tension der Bulbuskapsel auf die intraoculare Flussigkeitsbewegung von einschneidender Bedeutung ist und somit liegt in der Methode selbst eine Quelle des Misslingens, die durch zufällige Umstände wie Glaskörpereiterung noch ergiebiger werden kann.*)

Günstiger liegen in dieser Beziehung die Verhältnisse bei den subcutanen Injectionen. Aber auch hier kann ich die Bemerkung nicht unterdrücken, dass man in den Anforderungen an diese Methode und in Bezug auf die Grenzen der aus ihr zu ziehenden Folgerungen nicht zu weit gehen darf und sich namentlich hüten muss, auf die Schnelligkeit, mit der subcutan injicirte Flüssigkeiten im Auge sichtbar werden, zu viel Werth zu legen. Denn diese hängt in erster Linie von der Geschwindigkeit der Resorption im Unterhautzellgewebe $a b$ und letztere ist nicht nur bei verschiedenen Thieren eine verschiedene, sondern sie wechselt auch bei ein und demselben Thiere im Laufe der Zeit.

Als Beispiel führe ich ein Kaninchen an, das ich ohngefähr 2 Jahre lang besass und zur Demonstration der subcutanen Fluorescin-Injectionen zu benutzen pflegte. Mit der Zeit wurde das Thier unbranchbar, indem trotz gleichbleibender Quantität der Injectionsflüssigkeit die Erscheinungen im Auge ausblieben. Die Section zeigte den Grund. Es waren so zahlreiche subcutane Narben vorhanden, dass die Abhäutung sehr schwierig war und offenbar die Resorption des Fluorescin nur sehr mangelhaft erfolgen konnte. Einen ähnlichen Ein-

*) Auf Grund weiterer Erfahrungen rathe ich intraoculäre Injectionen direct durch die Bulbus-Insertion des Rectus superior porzunehmen; wie es scheint, kann man auf diese Weise das Ausfliessen des Glaskörpers und die Spannungs-Abnahme bis zu einem gewissen Grad verhüten. 
fluss mögen wohl Alter und verschiedene zufällige Umstände ausüben können.

Warum also in einzelnen Fällen die in Rede stehenden Experimente resultatlos bleiben können, dafür lassen sich Gründe im Allgemeinen entdecken und dieser Sachlage gegenüber muss der Kritiker wohl festhalten, dass hier, wie ja überhaupt, negative Resultate gegenuber positiven nicht in die Wagsehale fallen können.

Die bisher besprochene experimentelle Forschung ist nun aber nicht der einzige Weg, den man zur Entscheidung der Frage der intraoculären Flüssigkeitsbewegung einschlagen kann; gleichberechtigt sind die klinische Beobachtung, sowie die pathologisch-anatomische Untersuchung.

In dem Bestreben, diese ebenfalls nutzbar zu machen, wandte ich meine Aufmerksamkeit namentlich solchen Fallen zu, bei welchen krankhafte Processe im Fundus oculi sich discontinuirlich nach vorne propagirten.

Was ich in dieser Beziehung in der Litteratur, sowie durch eigene Beobachtung sammeln konnte, ist Folgendes:

Schon H. Müller*) war es aufgefallen, dass er bei Chorioiditis metastatica eine Ansammlung von Eiterkörperchen im ganzen Umfang des Canalis Petiti vorfand, obgleich der Process auf $1 / 3-1 / 4$ der Peripherie des Bulbus beschränlkt war.

Analoge Beobachtungen machten bei derselben Krankheit Hosch**), Heiberg***) und Andere.

Hosch wies in dem dem Canalis Petiti benachbarten Theil des Glaskörpers neben den Eiterkörperchen Mikrococcen nach, sowie ferner eine Anhäufung von Lymphzellen in der Peripherie der Iris, sowie im Fontaua'schen Raum.

Es fanden sich demnach dieselben Gegenden des Bulbus, die bei einer directen Tusehe-Injection von FarbstoffPartikelchen occupirt werden, von Eiterkörperchen gefüllt,

*) Gesammelte und hinterlassene Schriften.

**) Graefe's Archiv XXVI. 1, p. 189.

***) Centralblatt für d. med. Wissenschaft 1874, p. 561 . 
ohne dass diese Ansammlungen eine continuírliche Fortsetzung der eitrigen Chorioiditis bildeten.

Ich selbst war in einem Falle von Chorioiditis metastatica, den ieh anatomisch zu untersuchen Gelegenheit fand, nicht so glücklich wie die genannten Forscher. Zwar fand ich gleichfalls den vorderen Theil des Glaskörpers und den perilentalen Raum, sowie die Zonulafasern von Eiterkörperchen und Detritusmassen angefüllt, indess auch hintere und vordere Kammer waren von denselben Massen in einer Vollständigkeit gleichsam ausgegossen und das Irisgewebe in einer Weise eitrig infiltrirt, dass über die Provenienz dieser Massen ein Urtheil nicht mehr zu gewinnen war.

Etwas mehr Erfolg hatte ich in einem Fall von Glioma retinae, den ich bei einem Kinde von 2 Jahren in der Strassburger Augenklinik zu beobachten Gelegenheit hatte.

Aus dem Angeninnern kam der charakteristische gelbe Reflex; das Auge besass normale Tension, die Iris erschien normal, die Pupille erweiterte sich gut auf Atropin. Eigenthümlich waren perlartige, kleine, graue Kügelchen, die in der Anzahl von 6-10 für gewöbnlich am Boden der Vorderkammer lagerten, bei Augenbewegungen aber hin und her geschleudert wurden.

Schon bei der klinischen Betrachtung derselben vermuthete ich, dass man es mit Conglomeraten von Gliomzellen zu thun hatte und es erweckte mein lebhaftestes Interesse, auf welchem Wege diese Zellen in die Vorderkammer gelangt seien.

Die anatomisehe Untersuchung lehrte, dass die gliomatöse Neubildung auf den Fundus oculi beschränkt war und das Corpus ciliare nicht erreichte; trotzdem fand sich der perilentale Raum von Zellen infltrirt, die mit den Gliomzellen indentisch waren; zwischen den Processus ciliares, sowie zwischen den Fasern des freien Theils der Zonula und an der hinteren Fläche der Iriswurzel konnte man schon mit unbewaffnetem Auge solche Massen erkennen. Die Iris zoigte im Allgemeinen einen gewissen Zellenreichthum, namentlich aber zeichnete sich auf einer Reihe von Meridionalschnitten durch den vorderen Bulbusabschnitt dio Iriswurzel durch ZellenInfiltration aus und nicht minder war der Fontana'sche Raum 
und der Schlemm'sche Canal damit angefüllt. Dabei lag die Iris-Peripherie der Cornea dicht an.

Was die eigenthümlichen Kügelchen anbetrifft, so breiteten sie sich, auf ein Objectglas gebracht, flach aus, wie ein Regentropfen, wenn er auffällt und sie bestanden in der That aus Gliomzellen.

Aehnliche Beobachtungen von Fortbewegung von Gliomzellen aus dem Fundus oculi nach vorne scheinen auch sonst gemacht zu sein; so bemerkt z. B. Brailey *) bei Besprechung der Beziehungen zwischen Gliom nnd Spannungszunahme des Bulbus ausdrücklich ,,The ciliary folds and the lenscapsules are often separated by a broad stratum of glioma-cells."

Nun können allerdings solche Beobachtungen, wie die angeführten, nicht den Anspruch eines unanfechtbaren Beweises erheben: Lymphzellen and Gliomzellen sind eben ein zu wenig charakteristisches Material, namentlich für die Iris, wo ähnliche Zellen in wechselnder Menge normaliter vorhanden sind, als dass sie in gleicher Weise überzeugende Schlüsse gestatteten, wie etwa in Experiment das Vorhandensein von Tusche-Partikelchen oder die Färbung mit Berliner Blau. Aber wenn man sich das Gesammtbild vor Augen hält: ein Gliom- oder Eiterherd im fundus oculi in den Glaskörper hineinragend, eine Ansamm. lung von entsprechenden Zellmassen rings im vordern Glaskörper-Abschnitt, im perilentalen Raum, zwischen den Ciliarfortsätzen, hinter, in und vor der Iriswurzel, im Fontana'schen Raum und dem Canalis Schlemmii, so ist doch kaum zu leugnen, dass eine evidente Analogie mit der Localisation der Injectionsmassen, die experimentell in das Corpus vitreum injicirt werden, vorliegt, und ich meine, jeder Unbefangene wird anerkennen müssen, dass wenn man für diese Fälle die nämliche Erklärung heranzieht, ein Fortführen der pathologischen Massen auf intraocularen Strömungsbahnen von hinten nach vorn, dass dann den Thatsachen keinerlei Zwang angethan wird, ja

*) Ophthalm. Hosp. Reports X. p. 277 . 
dass diese Analogie von Experiment und pathalogischem Befund der in Rede stehenden Theorie eine gewisse Stütze verleiht. Den Einwurf freilich, dass hier pathologische Verhältnisse vorliegen, muss ich hier ebenso gut zulassen, wie ich stets betont habe, dass auch die Methode der directen Glaskörper-Injection nicht ganz davon freizusprechen sei.

Dieser Einwurf trifft auch den folgenden, meiner Ansicht nach werthvollsten Fall, den mir meine anatomischen Untersuchungen speciell zur Stütze der Frage der Irisfiltration geliefert haben.

August B., 11 Jahre alt, leidet an Hydrophthalmus congenitus; der Bulbus wurde wegen Schmerzhaftigkeit enucleirt; die eine Hälfte stand mir zur anatomischen Untersuchung zur Disposition.

Das Auge ist in allen seinen Durchmessern vergrössert, die vordere Kammer tief. Die Cornea rom Limbus her, namentlich in ihren äusseren Schichten vascularisirt und reich an Lymphzellen. In der Gegend der Corneo-Scleral-Grenze stark verdünnt $(0.4 \mathrm{Mm}$.), nimmt sie nach dem Centrum zu rasch an Dicke zu bis $0.9 \mathrm{Mm}$. Der periphere Theil derselben zeigt zwischen den einzelnen Hornhautlamellen auffallend grosse Spalten, namentlich in den der Kammer benachbarten Lagen. Die Lamellen selbst erscheinen wie gequollen, ebenso die Membrana Descemet.

Die Iris zeichnet sich durch ein sehr auffallendes Verhalten aus.

Der peripherste Theil derselben ist in der Breite von ca. $1 / 2 \mathrm{Mm}$. der Cornea stark genähert; auf manchen Schnitten liegt er derselben sogar an, ist aber nicht mit ihr verwachsen.

Diese Annäherung ist aber, im Profil gesehen, keine gleichmässige, sondern am stärksten an einer Stelle der Iris, die von der Kammerbucht etwa $1 / 2 \mathrm{Mm}$. entfernt ist. Hier steht die Membrana Descemet. mit der vorderen Irisfläche durch Vermittlung von etwas Exsudat und einer eigenthümlichen Structur-Veränderung der Iris in Contact.

Diese Structur-Veränderung besteht darin, dass die Iris an dieser Stelle in ihrer ganzen Peripherie gleichsam nach vorne ausgezogen ist, sodass ein ringförmig verlaufender Grat 
resp. auf dem Radiärschnitt eine kegelförmige Formation zu Stande kommt (Fig. 4).

Die Basis des Kegels entspricht der Hinterfläche der Iris, während die Spitze fast die Cornea berübrt. Innerhalb dieses Kegels zeigt nun die Irisfaserung die Eigenthümlichkeit, dass die Richtung der langgeschwänzten Zellen selbst kleinerer Gefässe eine zur Irisebene senkrechte ist, während zugleich eine leichte Convergenz nach der Spitze des Kegels, also nach der Membrana Descemeti Statt hat. Es ist ferner die ganze Iris namentlich der dem Grat entsprechende Theil, stark odematös, durchsetzt von einem in der Conservirungsflüssigkeit geronnenen Exsudat.

Dasselbe Exsudat findet sich hinter der Iris im Bereich der hinteren Kammer, sowie an ihrer Vorderseite an der Spitze des Kegels und von da peripher in der Kammerbucht. Diese letztere erscheint auf den Meridionalschnitten, auf welchen die Spitze des beschriebenen Iriskegels die Cornea berührt, in eine geschlossene Cyste verwandelt, die mit dem Exsudat gefüllt ist (Fig. 4).

Nachdem die Iris mit dem Grat des ringförmig auf ihrer Vorderfläche sich erhebenden Kammes die stärkste Annäherung an die Hornhaut vollzogen, weicht sie jäh, fast in rechtem Winkel nach hinten zurück, der Vorderfläche der Linse zu, mit der ein kleiner Theil des Pupillarrandes in Gestalt einer gewöhnlichen, hinteren Synechie verwachsen ist.

Das Corpus ciliare zeigt in der Gegend des Circul. Irid. maj. einen Herd von Lymphzellen. Die Processus ciliares sind so nach vorne gerichtet, dass sie fast der Iris parellel verlaufen, sie sind gleichfalls ödematös, sowie auch zwischen ihnen Exsudatmassen lagern. Ciliarmuskel und Gefässe zeigen nichts Besonderes. Der Canalis Schlemmii ist spaltförmig in die Länge gezogen, bisweilen durch Anlagerung seiner Wände geschlossen, arm an Zellen.

Der Glaskörper ist durchweg in der Müller'schen Flüssigkeit zu einer Gallerte erstarrt, in der sich Blutergüsse durch ihre braune Farbe markiren.

Die Retina ist zum grössten. Theil abgelöst, theils durch einen Bluterguss, theils durch ein seröses Exsudat, wie es auch den Glaskörper zu durchsetzen scheint.

Diese Netzhautablösung erstreckt sich übrigens viel weiter, als es makroskopisch den Anschein hat, indem sich mikrosko- 
pische Exsudatschichten fast überall zwischen Chorioidea und Pigmentepithel oder zwischen diesem und den ubrigen Netzhautschichten vorfinden. Die Stäbchen und Zapfen sind durchweg zerstört, die Körnerschichten arm an Körnern, dagegen von cystoiden Rüumen durchsetzt.

In der Chorioidea findet sich eine sogenannte Chorioiditis externa hämorrhagica wie sie von Goldzieher*) und Brailey beschrieben wurde.

Während nämlich die Chorio-capillaris keine auffälligen pathologischen Verändernngen zeigt (Die Grundsubstanz scheint namentlich in Anbetracht des kindlichen Alters des Patienten etwas trüber als normal), finden sich in der Schicht der grossen Gefässe und namentlich in der Suprachorioidea zahlreiche grosse und kleinere Blutergüsse neben serösen Exsudatmassen. Die Suprachorioidea ist stark hypertrophisch, sie lässt sich in sehr zahlreiche Blätter von dichtfaserigem Bau zerspalten, in welchen hier und da zartwandige Gefässe neben den normalen Zellen nachzuweisen sind. Das Blut findet sich zum Theil zwischen diesen Blättern in gut begrenzten, cystösen Räumen, zum Theil frei im Gewebe. Massenhaft ist ferner Hämatoidin vorhanden, theilweise von den Zellen der Suprachorioidea aufgenommen. An einigen grösseren Venen der Chorioidea fand sich eine starke Verdickung der Adventitia.

Die eine Arteria ciliaris longa, die ich untersuchen konnte, ist ca. von $5 \mathrm{mal}$ so starkem Kaliber als normal; in derselben findet sich die Intima elastica auf eine Strecke hin durch Blut unterwühlt. (Aneurysma dissecans.) blutleer.

Die Vasa vorticosa zeigen niclts Abnormes, sie sind

Ueber die Lage der Linse sowie über den Nervus opticus liess sich an der Bulbushälfte nichts Zuverlässiges ermitteln:

Was den Zusammenhang der einzelnen Punkte des anatomischen Befundes untereinander und mit der Formveränderung des Bulbus anbetrifft, so unterliegt es nicht dem mindesten Zweifel, dass eine so heftige und offenbar

*) C. Bl. f. Augenheilk. Bd. VII. p. 41.

**) Ophthalm. Hosp. Rep. X. p. 390 . 
chronisehe Chorioiditis wohl geeignet ist, einen Buphthalmus zu produciren.

Indessen in Anbetracht, dass es sich einsweilen nur um eine vereinzelte Beobachtung handelt - nur Braile ${ }^{*}$ ) erwähnt in einem Fall von Buphthalmus, dass er die Chorioidea hyperämisch, zellenreich und verdickt gefunden habe - beschränke ich mich auf diese Bemerkung, um die Aufmerksamkeit auf die eigenthümliche Structur- und Lage-Veränderung der Iris hinzulenken, die das lebhafteste Interesse erwecken muss.

Ich glaube nicht irre zu gehen, wenn ich sie als den nnzweideutigen Ausdruck einer durch die Chorioiditis exsudativa pathologisch gesteigerten Irisfiltration ansehe. Offenbar stammt all das Exsudat, das wir unter der Retina, im Glaskörper, im perilentalen Raum, in der eigenthümlich erweiterten hinteren Kammer und im Fontana'Raum vorfinden, aus der chronisch entzünđeten Chorioidea. Dass dies im Leben flüssige Exsudat aus der hinteren in die vordere Kammer gelangte, indem es die Iris quer durchfloss, dafür spricht sowohl der eigenthümliche Faserliauf an der nach vorne vom Exsudat ausgebuchteten Irisstelle als auch der Umstand, dass diese Stelle offenbar dem perilentalen Raum entspricht. Es ist ja bekannt, dass die buphthalmische Vergrösserung des Auges eine Dehnung der Zonula und eine Erweiterung des perilentalen Raumes mit sich bringt und damit stimmt es ;ut überein, dass wir die lrisfiltration weiter als normal pupillarwärts geruckt finden, sodass eben die Absackung der Kammerbucht zu Stande kommt.

Ich gestehe, dass mir eine andere Deutung, soviel ich darüber nachgedacht habe, ganz unmöglich erscheint.

Eine ringsum auf die Irisperipherie von hinten einwirkende Kraft muss vorhanden gewesen sein. Das ergiebt

*) Ophth. Hospit. Rep. X. p. 94. 
sich aus dem gleichmässigen Vorhandensein der Veränderung eben in der ganzen Peripherie der Iris.

Könnten die Processus ciliares vielleicht die Iris so nach vorne gedrängt haben?

Aber wie käme alsdann die Absackung des Fontanaschen Raumes, wie der eigenthümliche Faserverlauf an der betreffenden Irisstelle, zu Stande? Die Iris müsste im Gegentheil zusammengepresst erscheinen.

Oder sollen wir auf eine Entzündung im Fontana'schen Raum als Ursache recurriren? (Knies).

Aber es fehlt jede Spur einer auf diese Gegend beschränkten Entzündung und dann ist ja keine Iris-Synechie, sondern nur eine Anlagerung vorhanden und zwar eines Irisstücks, das von der Kammerbucht ca. 1/2 Mm. entfernt ist. Es giebt - ich wiederhole es - für den geschilderten Befund keine andere Erklärung, als eine Andrängung der Iris an die Cornea durch einen in der Abbildung ja als Exsudat sichtbaren, von hinten kommenden Flüssigkeitsstrom, der, indem er die Iris durchfliesst, sie der Stromrichtung nach ausfasert.

Von der grössten Wichtigkeit ist es nun, dass sich in der Literatur eine ganze Anzahl analoger Beobachtungen vorfinden. So hat Brailey*) Falle von Ablapselung der Kammerbucht bei primärem Glaucom beschrieben, die offenbar dem obigen analog waren. Die Kammerbucht war offen, dagegen lag die Iris mit einem mehr central dem Canalis Sehlemmii gegenüber gelegenen Punkt der Cornea an, ohne dass aber Verwachsung bestand. Gerade solche Falle waren es, welche Brailey veranlassten, die Knies'sche Lehre von der Entzündung des Fontana'schen Raumes zu verwerfen und sich für ein mechanisches Moment als Ursache der peripheren Iris-Synechie auszusprechen. Ich kann mir nicht versagen, Brailey's Ge-

*) 1. c. X. p. 15 u. 91 . 
danken über das Zustandekommen der Synechie hier wörtlich wiederzugeben, weil sie der unmittelbare Ausfluss der anatomischen Anschauung waren, die auf einem ungewohnlich reichen Glaucommaterial basirte und weil sie zu einer Zeit geäussert wurden, wo die Frage der Irisfiltration noch nicht auf der Tagesordnung stand.

"The cause of this application of the periphery of the iris to the rim of the aqueous chamber seems to be an increased flow of fluid from behind the plane of the lens, issuing some where between the ciliary muscle and the periphery of the lens, for, as before said in aphacic cases, where the communication is widely opened and in subacute cases, where the flow is shight, this condition does not exist.

It is clear the cause is a mechanical one from the case in which the iris is applied but not adherent and I do not think that the advance of the lens will account for it. That the iris seems not to allow the passage of fluid through it, from its posterior to its anterior surface, seems to derive confirmation from the fact, that when the iris prolapses into a corneal perforating wound, this ceases to leak if the iris is large enough to fill it completely and we have anterior staplyloma following; and also from the bulging forwards of the iris between its pupillary edge and its periphery, frequently seen with exclusion of the pupil. In the same way I think the increased pressure bulges the iris forwards in glaucoma."

Aus dieser Darlegung geht hervor, dass Brailey nicht nur den von mir experimentell zur Anschauung gebrachten Flüssigkeitsstrom rings im perilentalen Raum gegen die Hinterfläche der Iris annimmt, sondern dass die unbefangene Betrachtung des anatomischen Bildes ihm sogar den Gedanken der Irispassage nahe legte. Wenn er diesen Gedanken schliesslich wieder verwarf, so verlohnt es vielleicht der Mühe die Gründe zu prüfen, die ihn dazu führten.

Weil bei Irisprolaps die Vorderkammer sich wiederherstellt und die vorgefallene Iris nicht continuirlich das 
Kammerwasser durchsickern lässt, deshalb, meint Brailey, sei eine Filtration durch die Iris auszuschliessen.

Zunächst ist dazu zu bemerken, dass es Fälle von Iris-Prolaps gibt, in denen Wochen lang die Kammer aufgehoben bleibt, aber in der überwiegenden Mehrzahl der Fälle ist es allerdings richtig, dass der Prolaps die Hornhautöfnung verschliesst. Nun hätte Brailey nur in einem einzigen Fall die enzündlichen Veränderungen, die Verdichtung und Verdickung des Gewebes, die sich an einer in eine Hornhautwunde eingestülpten und gefalteten Iris vollziehen, untersucht. so würde er wohl kaum dies Gewebe als Beweis für die Filtrationsunfähigkeit der normalen Iris herangezogen haben; noch weniger wird es Jemand in den Sinn kommen, das sclerotische Gewebe, aus dem die Iris bei chronischer Iritis besteht, zam Vergleich zu wählen, wenn er nur einen Blick auf die Abbildung wirft, die ich von einer solchen, Gräfe's Arch. XXVIII, 2 Taf. IV, Fig. 2, gegeben habe. Eine solche Iris verhält sich in Bezug auf Filtration zur normalen etwa wie Pergament zu Fliespapier.

Von einer Structurveränderung der Iris da, wo dieselbe gegen die Cornea hingedrängt ist, erwähnt Brailey nichts; dagegen finden sich sehr vollständige Angaben in dieser Beziehung bei Schnabel in seinen Beiträgen zur Lehre vom Glaucom.*)

In einem Fall (1) von Secundärglaucom nach Leucoma adhaerens beschreibt Schnabel die Tris wie folgt: „Das Ursprungsstück der Iris ist dicker als normal. Dies Verhalten erklärt sich nicht aus einer wahrhaften Zunahme der Masse, sondern durch Auseinanderziehung derselben, was sich schon daraus erkennen lässt, dass die pigmentirten Zellen in dem betreffenden Stück eine abnorme Richtung bekommen, indem die langen

*) Arch, f. Augen- u. Ohrenheilkunde von Knapp und Moos. Bd. VI. p. 129 . 
Fortsätze derselben gegen die Cornea resp. die hintere Irisfläche gerichtet sind."

Ferner in einem andern Fall (2) von Leucoma adhaerens: „An manchen Stellen war die Vorderfläche der Tris in einen Zipfel aufgehoben, der sich in eine Einsenkung am Rande der Descemet. Membran hineinlegte und daselbst festgehalten war. Gleicherwärts von dieser Verlöthungsstelle erschien dann die Iris wieder entweder ganz frei oder es zogen einzelne Bänder vom Ligam. pectinatum zur vordern Fläche der Iris." Weiter unten: „In diesen Schnitten war die Iris der Cornea soweit genähert, dass die Vorderfläche ihres Ursprungsstückes mit der Scleralfä.che des Ciliarmuskels in eine Ebene zu liegen kam und gegenüber dem Rand der Descemet. Membran bestand eine spitzige Erhebung im Irisprofil, welche die Cornea aber nicht erreichte."

Endlich noch in einem dritten Fall (12 p. 143): „Das Gewebe des betreffenden (peripheren, verlötheten) Irisstückes war sehr stark aufgelockert, die vordere Fläche gleichsam aufgehoben und festgehalten, ohne dass die hintere Fläche eine wesentliche Lageveränderung eingegangen hätte."

Schnabel's exacte Beschreibung macht is auch ohne Abbildung überzeugend sicher, dass in seinen Fallen von Secundär-Glaucom dieselben Iris-Veränderungen vorlagen, wie sie meine Abbildung (Fig. 4) veranschaulicht. Uebrigens macht er an anderen Stellen auch besonders noch darauf aufmerksam, dass bisweilen die Kammerbucht dadurch geschwunden war, dass die Iriswurzel sich der Cornea sehr stark näherte, ohne indess eine Verwachsung einzugehen.

Auch speciell für Buphthalmus findet sich in der Literatur eine Beobachtung ron $\mathrm{Raab}^{*}$ ), die vielleicht hier anzuführen wäre.

In einem Fall von Hydrophthalmus congenitus fand sich, neben Linsenluxation, in der Iris eine der in Rede stehenden ähnliche Veränderung nur an einer mehr central gelegenen Stelle, vielleicht entsprechend der partiellen Verbreiterung des

*) Klin. Monatsblätter für Augenheilkunde 1876. 
perilentalen Raumes durch die Linsen-Luxation. Die Beschreibung ist leider in dieser Reziehung nicht hinreichend genau, um ein Urtheil zu ermöglichen. „Etwa in der Mitte zwischen Pupillarrand und Insertion erhob sich aus dem Irisstroma ein von Gefässen durchzogener Zipfel, der bis an die hintere Corneaflache reichte, aus welcher gleichfalls neugebildetes Gewebe hervorsprosste, wodurch die Iris festonartig nach vorne gezogen wurde."

Ich komme nun zum Schlusse dieses Abschnittes. Was eigene und fremde Beobachtung auf dem Gebiet der experimentellen, der klinischen und anatomischen Untersuchung mir an geeignetem Material boten, um für die Beantwortung der Frage der Irisdurchquerung durch das Kammerwasser verwandt zu werden, dus habe ich mich bemuht, objectiv darzulegen; es erubrigt nur noch, Einiges vom allgemeinen physicalischen Standpunkt hinzuzufügen.

Viele der angeführten Thatsachen deuten darauf hin, dass der Weg, den das Kammerwasser aus der hinteren in die vordere Kammer einschlägt, vom intraocularen Druck abhängig ist. Dieser Einfluss scheint mir darauf $\mathrm{zu}$ beruhen, mit welcher Innigkeit Iris und Linse durch denselben aufeinander gepresst werden. Ist die Tension des Bulbus gleich Null wie nach Paracentese der Cornea, so bilden hintere und vordere Kammer gleichsam einen einzigen Flüssigkeitssee, der sich um den Pupillarrand herumwindet, ist dagegen normale oder erhöhte Tension vorhanden, welch letztere in der Regel mit Zufluss-Vermehrung einhergehen wird, so wird der Verbindungsweg zwischen Linse und Iris geschlossen, obne dass man dabei an einen hermetischen Verschluss zu denken hat. Die Communication findet jetzt quer durch die Iris statt, weil bei normaler Structur derselben dieser Weg der kürzeste und leichteste ist. Liegt darin nun eigentlich etwas Befremdliches?

Alle Anatomen, welche die Iris beschreiben, nennen das Gewebe derselben schwammig, spongioss, manche ver- 
gleichen es geradezu nit einem Schwamm, der bei der wechselnden Pupillen-Contraction abwechselnd Flüssigkeit einsaugt und auspresst. Ist es nun wirklich so merkwürdig, dass unter diesen Umständen eine FlüssigkeitsCommunication durch diese Membran stattfindet und wäre es im Gegentheil nicht auffallend, wenn das Kammerwasser längs der hinteren Irisfläche einherflösse, als wäre diese mit Firniss bestrichen?

Würde Jemand ein Augen-Phantom verfertigen und die spongiöse Iris z.B. aus dickem Fliespapier nachbilden, würde das Kammerwasser an dieser künstlichen Iris einherfliessen ohne in sie einzudringen? Gewiss gestalten sich ja für die lebende Iris, die von Pigment und Glashaut bekleidet ist, die Verhältnisse der Filtration complicirter, aber es ist doch gut, an das grob Mechanische und Einfachste dabei zu erinnern.

Dass es für die Communication beider Kammern. einen Unterschied macht, ob der Bulbus so weich ist, dass Linse und Iris haltlos bei Augenbewegungen schlottern, oder so hart, dass Iris und Linse bei Glaucom fest auf einander gepresst werden und die Vorderkammer auf ein Minimum reducirt ist, das wird doch wohl kein Unbefangener bezweifeln, und so gut die Ernährung der Retina von der Chorioidea aus durch die Glashaut und das Pigmentepithel hindurch stattfindet, so gut als die complicirt gebaute Gefässwand den Flüssigkeits-Durchtritt gestattet, so gut erscheint mir auch die Möglichkeit der Irisfiltration zuzulassen.

Noch in einer andern Richtung ist das beigebrachte Material verwendbar.

Einem Ophthalmologen von Fach wird es ja wohl kaum beikommen, daran zu zweifeln, dass die Hauptquelle des Humor aqueus in dem Corpus ciliare zu suchen sei; aber damit scheint es mir ganz gut vereinbar, dass auch aus dem Glaskörper eine Beimengung von Flüssigkeit 
erfolgt. Das scheint mir nicht nur a priori wahrscheinlich, sondern es sprechen doch auch manche pathologischen Befunde und die meisten Versuchsergebnisse, unter andern z. B. auch die von Deutschmann an menschlichen Leichen erhaltenen, für diese Annahme.

II. Bindegewebs-Sclerose der Iris.

Soviel abweichende Ansichten ther die Entstehung des Glaucom im Laufe der Jahre auch geăussert wordeǹ sind, uber einen Punkt der Glaucomlehre war man namentlich seit A. v. Gräfe stets einig, dass nămlich eine bestimmte Classe Secundär-Glaucome sich an entzündliche Vorgänge in der Iris anschlösse.

Diese Beobachtung musste vom Standpunkt der Irisfiltration ein neues Interesse gewinnen und war die Veranlassung, dass ich das Irisgewebe bei vordern und hintern Synechien einer anatomischen Untersuchung unterzog.

Die Resultate, die ich bereits publicirt habe*), lassen sich kurz dahin zusammenfassen, dass das im normalen Zustande sehr spongiöse Gewebe der Iris eine mehr oder weniger fibrose Beschaffenheit annimmt und dass an den Gefässen sclerotische Wandveränderungen auftreten, die in vielen Fällen zum Verschluss derselben, oft durch pigmentirte Thromben, führen.

Die jenen Untersuchungen beigegebene Tafel IV, Fig. 2 u. 3, geben ein Bild, bis zu welehem Grade diese Verdichtung des Gewebes gelangen kann und doch ist damit noch nicht das Maximum erreicht.

In denjenigen Fällen von Leucoma adhaerens, die zu Staphyloma corneae führen, kommt es häufig zu einer so hochgradigen Atrophie der Iris, dass von ihr fast nur noch eine Pigmentlage übrig bleibt, welche die Membrana Decemet. bekleidet.

*) Graefes Archiv Bd. XXVI. p. 35.

v. Graefe's Archiv für Ophthalmologie, XXX. 4. 
In derselben Arbeit babe ich auch über Untersuchungen berichtet, die Irides betrafen, welche von Glaucoma inflammatorium oder absolutum stammten. Zum grössten Theil wurde das Material bei Iridectomieen gewonnen, zum kleinsten Theil standen mir die ganzen Bulbi zur Disposition. Die gefundenen Gewebsveränderungen waren analog denen bei Secundär-Glaucom. Indess gerade deshalb war der Verdacht nicht von der Hand zu weisen, dass wir es hier gleichfalls mit secundären Veränderungen zu thun hätten, Veränderungen, die sich im Verlaufe des glaucomatösen Processes erst $\mathrm{zu}$ diesem hinzugesellten, vielleicht sogar durch die complicirende Entzündung vermittelt würden.

Um mich hiergegen sicher zu stellen, wandte ich in den letzten Jahren meine Aufmerksamkeit der Untersuchung einfacher Glaucomfälle zı oder aber solcher Fälle von Glaucoma inflammatorium, bei denen die begleitende Entzündung so jungen Datums war, dass auf sie eine etwa gefundene Iris-Sclerose nicht wohl bezogen werden konnte. Das Material dazu lieferten mir wieder die bei Iridectomieen gewonnenen Irisstückchen.

Der Gang der Untersuchung brachte es nun mit sich, dass ich häufig auf den Vergleich mit normaler Iris recurriren musste. Denn da die glaucomatöse Iris ausnahmslos senilen Augen entstammte, so war ja stets die Möglichkoit vorhanden, dass vorgefundene anatomische Veränderungen einfach seniler Natur seien und diese Möglichkeit machte es mir zur Pflicht gerade die senilen Iris-Veranderungen zum Gegenstand besonderen Studiums zu machen.

Es hat sich dabei ergeben, dass in der That $z$ wischen der infantilen und senilen Iris sehr wesentliche Differenzen existiren und zwar beziehen sich dieselben namentlich auf den Bau der Gefăsse und Nerven. 
Bei älteren Individuen verhalten sich die Gefässe folgendermassen :

Die Arterien besitzen eine sehr entwikelte bindegewebige Adventitia. Die Bindegewebsfasern derselben verlaufen im Allgemeinen parallel der Gefässrichtung, doch so, dass die einzelnen Züge sich bisweilen spiralig um das Gefäss herumwinden; ausserdem giebt es im innersten Theil der Adventitia, der an die Muscularis anstösst, wirklich circulär verlaufende Fasern. Die Adventitia ist bei älteren Individuen arm an Zellen, dagegen nach aussen von einem Netz anastomosirender Zellen umsponnen.

Aus diesem Verhalten der Adventitial-Scheide erklärt sich ungezwungen das Aussehen eines Querschnittes, wie ihn Fig. 5 wiedergiebt.

Auf die am weitesten nach aussen gelegenen umspinnenden Zellen folgt eine punktirte Zone (die Querschnitte der Längsfasern) und anf diese der Ring der circulären Fasern.

Trifft indess ein solcher Querschnitt zufällig die Ebene einer stark gewrudenen Spirale, so kann es vorkommen, dass die Adventitia nur oder fast nur aus circulären Fasern zu bestehen scheint.

An die Adventitia schliesst sich nach innen die Muscularis. Dieselbe besteht nur ans Ringmuskelfasern, die vielleicht mit elastischen Fasern gemischt sind. Sie ist selbst bei den grössten Iris-Arterien spärlich entwickelt (vergl. Fig. 6) und wird noch spärlicher in dem Maasse als die Arterien kleiner werden, so dass auf Quersehnitten mittlerer und kleiner Gefässe häufig ihr Charakter, $a b$ Arterie oder Vene, nicht zu bestimmen ist.

Der Ringmuseulatur folgt unmittelbar die Intima elastica, sehr dunkel und seharf gezeichnet; sie erscheint in der gewöhnlichen Weise längs gestreift und ist bekleidet von dem Gefässendothel, an dem namentlich die Kerne 
durch eine exquisite Stäbchenform auffallen (Fig. 6). Die Länge derselben beträgt ca. 0,014 Mm., ihre Breite ca. $0,002 \mathrm{Mm}$. Ich habe sie früher für die Kerne einer nach innen gelegenen Längsmusculatur gehalten *), bin aber mittlerweile von dieser Ansicht zurückgekommen.

Man kann sich nämlich an den Arteriis ciliar. long. direct, dadurch, dass man sie aufschneidet und von inņen betrachtet, aberzeugen, dass auch bei ihnen die Kerne des Gefassendothels auffallend schlank sind, wie das schon H. Muller gesehen hat, während an den grossen Venen der Chorioidea dieselben Kerne dicker und kürzer erscheinen. Dasselbe Verhältniss erhält sich nun auch für die Iris, sodass darin ein gutes Unterscheidungsmerkmal für Arterien und Venen gegeben ist.

Was die letzteren anbetrifft, so verhalten sie sich in Bezug auf die Adventitia wie die Arterien; dagegen ermangeln sie jeglicher Muscularis. Es folgt auf die Adventitia sofort die Intima und darauf das durch ovale Kerne ausgezeichnete Endothel. Häufig sieht man der Aussenseite der Intima platte Zellen anliegen, so dass dieselbe einen doppelten Contour erhält.

Auch die Capillaren besitzen eine verhältnissmässig starke Adventitia, die nach aussen durch spärlicher vertretene umspinnende Zellen abgegrenzt ist. Das Capillarrohr selbst besitzt nach aussen einen Belag platter Zellen, die sich deutlich von den die Capillarwandung constituirenden Zellen unterscheiden lassen; die letzteren haben ovale Kerne.

Bei Kindern sind nun alle diese Verhaltnisse bei weitem schwieriger zu übersehen und zwar zunächst, weil sowohl das die Adventitia umspinnende Zellennetz als aucb diese selbst einen Reichthum an Zellen aufweist, der p. 255 .

*) Bericht der Naturforscher-Versammlang zu Freiburg 1883. 
die Erkennung des nach innen liegenden Gefässrohres sehr ersehwert.

Dazu kommt, dass das Bindegewebe der Adventitia ausserordentlich zartfaserig ist und einen dichten Filz bildet, in dem eine Faserrichtung schwer zu erkennen ist. So kommt es, dass auf Gefässquerschnitten der Adrentitia gewöhnlich die äussere punktirte Zone fehlt, dieselbe erscheint vielmehr, wenn auch undeutlich, circulär gezeichnet und zugleich so zellenreich, dass die Grenze gegen die Muscularis schwer zu ziehen ist (Fig. 7). Die Intima elastica ist bei Kindern bei weitem weniger scharf als Contour markirt, als bei älteren Personen.

Wenn die geschilderten Verschiedenheiten im Bau der Gefässe bei dem grossen Reichthum der Iris an solchen schon allein genügen, für die ungefăhre Altersbestimmung einen Anhalt zu liefern, so trägt das Verhalten der Nerven in noch auffallenderer Weise dazu bei.

Bei alteren Personen gelingt es mit Leichtigkeit, namentlich im peripheren Theil der Iris durch Behandlung mit Osmiumsäure zahlreiche markhaltige Nerven zur Anschauung zu bringen; man erhält gelegentlich Schnittserien meridionaler Richtung durch Corpus ciliare und Iris, wo fast Schnitt für Schnitt eine Nervenfaser aufweist, die in bemerkenswerther Nähe am Circul. Irid. major vorbeistreicht nnd sich in die Iris begiebt; aber auch im pupillaren Theil derselben trifft man markhaltige Nerven, wenn auch ausnehmend spärlich. Sie verlaufen einzeln oder $\mathrm{zu} 2$ bis 4 und sind gewöhnlich von Zügen starkfaserigen Bindegewebes begleitet. Doch trägt diese Begleitung fast nie den Charakter einer eigentlichen regelmässig gebauten Hülle, wie dies z. B. bei der Gefäss-Adventitia der Fall ist; vielmehr erscheinen die Nervenfasern gewöhnlich an diese Bindegewebsbündel eher wie angelehnt; davon überzeugt man sich auch an Querschnitten. 
Nun habe ich eine ganze Reihe Irides von Kindern in den ersten Lebensjahren untersucht, ohne dass es mir gelungen wäre, auch nur eine einzige markhaltige Nervenfaser aufzufinden, trotzdem die Irides der gleichen Osmiumbehandlung unterzogen waren wie die der Erwachsenen. Ich kann demnach, angesichts der Leichtigkeit, mit der die Osmiumbehandlung die Nervenfasern zur Anschauung bringt, nicht anders als glauben, dass die Regenbogenhaut kindlicher Individuen markhaltige Nervenfasern nicht besitzt. Aber noch mehr.

Verfertigt man bei älteren Individuen Querschnitte der Iris nicht in radiärer, sondern dazu senkrechter Richtung, etwa aus der Mitte zwischen Pupillarrand und Irisinsertion, so trifft man ausser den zahlreichen Querschnitten der radiären Irisgefăsse, meistens mehr der vorderen Irisfläche zu gelegen, eine Reihe von Querschnitten, die auf den ersten Blick ebenfalls als Gefässe imponiren. (Fig. 8).

Zunächst nach aussen ein Ring umspinnender Zellen, dann eine punktirte Zone, die häufig nach innen etwas dunkler erscheint und darin, aber durch keinen scharfen Contour abgegrenzt, ein lichtes Centrum, meistens ganz homogen, in der Mitte dieses Centrums alsdann ein Punkt oder anch kleiner Kreis bisweilen etwas excentrisch gelegen, bisweilen ganz fehlend: so stellt sich im Allgemeinen dieses Pseudo-Gefäss dar. Die Gefässälnnlichkeit würde noch frappanter sein, wenn sich nicht der centralgelegene Punkt bei verschiedener Einstellung des Mikroskops als centralverlaufende Faser entpuppte.

Um über die Natur dieser Gebilde ins Klare zu kommen, nahm ich zunächst meine Zuflucht zu Flächenschnitten der Iris. Dieselben erfullten dann auch insofern meine Erwartung, als sie röhrenähnliche Bindegewebs-Stränge zeigten, die offenbar dem Querschnitt Fig. 8 entsprachen. Fig. 9 gibt eine wohlgelungene Abbildung, die mich der weiteren 
Beschreibung überhebt. Die axial verlaufende, in diesem Fall ausserordentlich feine Faser, zeigt hier und da Anastomosen, kleine Anschwellungen, bisweilen einen anliegenden Zellkern oder anliegende feinste Pigmentpünktchen. In anderen Fällen war die Faser etwas dicker, leicht streifig, auch sah ich einmal sowobl den ganzen Strang, als die centrale Faser eine Theilung eingehen. Wie auf Flächenschnitten, so liess sich dies Verhalten auch auf Zerzupfungspräparaten in gleicher Weise constatiren.

An der Gefäss-Natur dieser Gebilde fest zu halten, schien hiernach kaum möglich. Dagegen sprachen ferner anch die Gefässinjectionen der Iris, die mit Berliner Blau von der Arteria ophthalmica aus vorgenommen wurden, niemals enthielten die fraglichen Röhren eine Spur der Injectionsmasse.

Anderseits war es unverkennbar, dass der feinere Bau der axialen Faser stark an marklose Nervenfasern erinnerte.

Die Goldchlorid-Behandlung wurde zur Entscheidung angerufen, aber sie versagte. Dadurch, dass auch die Gefassintima das Goldchlorid stark reducirte, wurde der Werth des Verfahrens für die Diagnose der fraglichen Nervenfasern illusorisch.

Trotz alledem bin ich nach reiflicher Erwägung des pro und contra zu der Ansicht gelangt, dass wir in den in Rede stehenden Gebilden höchst wahrscheinlich marklose Nervenfasem zu sehen haben, die, umspült von Lymphe, in einer starken Bindegewebsscheide verlaufen: eine Eigenthümlichkeit, die wohl mit der nie ruhenden Pupillenbeweglichkeit in Zusammenhang steht, die es aber allerdings unter Umständen sehr schwierig machen kann, namentlich Querschnitte derselben von Querschnitten obliterirter Gefässe zu unterscheiden.

Merkwürdiger Weise ist es mir nun trotz aller Bemühung nicht gelungen, in der infantilen Iris diese feinen Nerven mit ihrer Hülle wiederzufinden; man sieht wohl 
hier und da auf Flächenschnitten sehr zellenreiche Bindegewebsstränge, ähnlich der Gefässadventitia, in denen kein Gefässrohr zu entdecken ist; aber ebensowenig vermochte ich jemals in denselben jene feinen axialen Fasern zu sehen. Das ist um so auffallender, als wir auch schon die markhaltigen Nervenfasern in der Iris des Kindes vermissten, und da es unmöglich erscheint, dass die kindliche Regenbogenhaut überhaupt keine Nerven besitzt, so liegt der einzige Ausweg in der Annahme, dass bei dem Zellenreichthum der kindlichen Nerven-Scheide und der Feinheit der axialen Faser diese der Wahrnehmung entgeht.

Im Uebrigen hätte ich zu der normalen Anatomie der Iris nur noch eine Bemerkung zu machen.

Wenn Michel in der vorderen Stromaschicht der Iris die Bindegewebsfibrillen vollständig fehlen lässt, so kann ich diese Ansicht in diesem Umfang nicht gelten lassen. Freilich kommt das reticuläre Aussehen dieser Schicht durch die Anordnung der verschiedenartigen Zellen mit ihren Ausläufern zu Stande; aber wenn man ganz frisch mit Osmium behandelte Iris zur Untersuchung verwendet, so sieht man, dass nicht nur überall in den Lücken dieser Schicht, sondern auch, allerdings spärlicher, in der Gefässschicht ein Gewirre von ausserordentlich zarten Bindegewebsfibrillen vorhanden ist, das gleichsam als Ausfüllungsmasse dient. Es sind dieselben allerdings unvergleichlich feiner als die Fibrillen des Bindegewebes, das die Gefässe und Nerven begleitet.

Wenden wir uns nun der Schilderung der pathologischen Iris-Veränderungen bei Glaucom zu, so wird es sich, um zunächst ein scharf gezeichnetes Bild derselben zu erhalten, empfehlen, von einem späteren Stadium der Krankheit auszugehen.

Was an der Iris am meisten auffallt, ist die grosse Dichtigkeit des Gewebes; der normale spongiöse Bau ist vollständig verloren gegangen. Es beruht dies zum Theil 
auf einer Schrumpfung des Gewebes; denn man kann allgemein behaupten, dass die Dicke der Iris bei Glaucom auf die Hälfte der normalen oder noch weniger reducirt ist, zum Theil kommt sie zu Stande durch die Selerosirung der Bindegewebsfibrillen, sowohl derer, die das feine Gewirre in den Stromalücken zusammensetzen, als der gröbern, welche die Gefässe und Nerven begleiten und die Septa zwischen den einzelnen Abtheilungen des Sphineter iridis bilden.

Das feine, unter normalen Umständen schwer sichtbare Bindegewebe erscheint in der solerotischen Iris gleichsam als eine dichte Grundsubstanz, in der die Zellen der Iris in grosser Dichtigkeit eingelagert sind; die letzteren selbst sind vorwiegend Spindelzellen mit kurzen Ausläufern oder Lymphzellen.

Gröber und mehr in die Augen fallend sind die sclerotischen Veränderungen der Gefäss-Adventitia.

Wie Fig. 10 zeigt, ist das Frappanteste an ihr ihre glänzende helle Farbe, die sich aus der zellenreichen und stark pigmentirten Umgebung doppelt deutlich hervorhebt, und der gänzliche Mangel derselben an Zellen. Dabei kann die Muscularis sowie die Intima noch erhalten sein, das Gefäss kann Blut führen, selbst dilatirt sein oder aber es kann dasselbe seine Wegsamkeit eingebüsst haben. Das letztere kommt entweder dadurch zu Stande, dass das Lumen mechanisch zusammengepresst erscheint oder dass es thrombosirt ist; ich sah einmal einen feinkornigen farblosen Thrombus, der verhältnissmässig frisch schien, einmal einen solchen, der eine deutliche Pigmentirung zeigte, sonst aber stets nur Thromben, die aus reinem Pigment zusammengesetzt waren (Fig. 11).

Was die einfache Verlegung der Gefässe durch Zusammenpressen betrifft, so findet man in der Iris bei Glaucom ungemein häufig Gefässquerschnitte mit spaltoder punktförmigem Lumen; ist das letztere der Fall, so 
wird die Diagnose durch das eigenthümliche, oben beschriebene Aussehen der Nervenquerschnitte, die versehlossenen Gefässen sehr ähnlich sehen, erschwert, und man ist häufig ausser Stande, zu sagen, ob man in einem bestimmten Falle überhoupt ein Gofäss vor sich hat. Indessen einerseits fällt bei Glaucom die Häufigkeit der fraglichen Gefässquerschnitte auf und anderseits kann man auf Flächenschnitten sich meist vor Irrthümern schützen.

Wenn man Gefässe in längerem Verlauf untersuchen kann, überzeugt man sich, dass an einem und demselben Gefäss Dilatation, bisweilen von sackartiger Form, mit Verengerung resp. Verschluss abwechselt, und bietet es der Zufall, dass man bei einer Windung des Gefässes Längs- und Querschnitt zugleich sieht, so erkennt man auch, welch grosse Aehnlichkeit ein einfach verschlossenes Gefäss und jene Nerven mit ihrer starken Adventitia auf Querschnitten besitzen. Bei den Pigmentthrombosen der Gefässe tritt bisweilen insofern eine Schwierigkeit ein, als auch die Wand und die Umgebung des Gefässes derart von Pigment infiltrirt sind, dass das Gefäss kaum deutlich zu isoliren ist.

Diese starke Pigmentirung der Iris bei Glaucom, die ihr, selbst wenn man das hintere Pigmentblatt entfernt hat, schon makroskopisch einen durchaus brännlichen Farbenton verleiht, ist, soweit ich gesehen, etwas sehr constant Vorhandenes und namentlich in der Sphinctergegend und im Ciliartheil ausgesprochen; den letzteren sah ich verhältnissmässig häufig auf Radiärschnitten in Form eines pigmentirten Stranges an die hintere Fläche der Hornhautperipherie angepresst, so dass gerade hier Pigmentthrombosen der Gefässe mit Sicherheit schwer zu diagnosticiren sind.

Nun sind aber neben diesen Verengerungen and Verstopfungen von Gefässen anderseits zahlreiche Dilatationen 
vorhanden. Nicht nur dass einzelne radiär verlaufende Irisgefässe eine nicht zu verkennende Erweiterung erfahren, so ist es namentlich wieder der Pupillartheil der Iris, der ja oft schon im Leben auf seiner Vorderfache in die Vorderkammer vorspringende Convolute von varicösen Gefässen erkennen lässt, wo dann mikroskopisch enorme Dilatation der kleinen Gefässe neben rerhältnissmässig umfangreichen Verodungen und Verstopfungen gefunden werden.

Um ein Urtheil über den Umfang dieser Gefäss-Veränderungen und dadurch ein Urtheil über die resultirenden Circulationsstörungen zu gestatten, war ein Fall von doppelseitigem Glaucom sehr geeignet, bei dem icb Gelegenheit hatte, das eine Auge von der Arter. ophthalmica aus, das andere (partiell) von der Arteria ciliar. long. externa mit Berliner Blau zu injiciren. Ein glücklicher Zufall hatte ihn mir, als angeblich normal, in die Hände gespielt zu der Zeit, wo ich damit beschäftigt war, an injicirten Augen die senilen Gefässveränderungen der Iris zu studiren. Erst die anatomische Untersuchung ergab, dass Glaucom vorlag und durch Nachforschen bei den Angebörigen der Verstorbenen konnte ich feststellen, dass dieselbe zwei Jahre vor ihrem Tod von Herrn Dr. Roder in Strassburg behandelt worden war. Ich verdanke der Freundlichkeit desselben die nachfolgenden klinischen Notizen.

Rosalie C., 76 Jahre alt, war seit Jahren bettlägerig; die Untersuchung der Augen musste deshalb in ihrer Wohnung und im Bett vorgenommen werden; es wurde sich auf die Stellung der Diagnose beschränkt.

R. Glaucoma absolut.

L. Glancoma subacut. $\mathrm{S}=$ Finger ca. $\mathbf{1}^{1 / 2}-2^{\prime}$, Cornea sehr trüb, Vorderkammer eng.

L. Breite Iridectomie, nach innen; die Heilung erfolgte sehr zögernd, die vordere Kammer stellte sich nur langsam wieder her; doch resultirte schliesslich eine Besserung des Sehvermögens, die aber nur vorübergehend war. 2 Jahre nach 
der Operation, als die Patientin starb, besass das Auge noch Lichtschein.

Die anatomische Untersuchung ergab, kurz referirt, Folgendes:

Das rechte Ange wurde von der Arter. ophthalm, aus mit Berliner Blau injicirt.

Die vordere Kammer sehr eng; periphere vordere IrisSynechie von beträchtlicher Breite. Die Dicke der Tris beträgt ca. $1 / 2$ so viel als normal, die Pupille über mittelweit, di Breite der Iris schwankt zwischen 1 und 1.2 Mm. Die Farbe der Iris, nach Entfernung des Pigmentblattes, braungelb, die Linse cataractös getrübt.

An der inneren Seite des Bulbus findet sich längs des Verlaufes der Arter. ciliar. longa ein Scleral-Staphylom, im Bereich desselben ist die Sclera verdünnt, die Chorioidea atrophisch, pigmentfrei und mit der Sclera verwachsen. Die Arteriae ciliares longae sind dilatirt, das Lumen der ausseren beträgt $0.056 \mathrm{Mm}$. im Durchmesser, die Dicke der Wand $0.012 \mathrm{Mm}$.

Die Retina ist windenblüthenartig abgelöst; innerhalb derselben findet sich eine gelatinöse Masse mit einigen Blatungen. Der Sehnerv, an dessen Papille die Retina stielförmig anhaftet, ist vollständig atrophisch, eine Excavation ist nicht zu constatiren, selbst die Lamina cribrosa zeigt sich nicht concaver als gewöhnlich.

Von der Iris wurde ca. $1 / 4$ zu mikroskopischer Untersuchung verwandt, die anderen $3 / 4$ abgelöst, mit Terpentinöl aufgehellt und bei schwacher Vergrösserung in toto untersucht.

Die sclerotischen Veränderungen der Iris waren höchst ansgesprochen, so war z. B. der Sphincter iridis nebst vielen in den Bindegewebssepten desselben verlaufenden Gefässen durch die Sclerose des Gewebes vollständig untergegangen, sodass das ganze Pupillarende der Iris eine verhältnissmässig homogene, glänzende Masse schien. Die Pigmentirung, die Gefässveränderungen sind durch Fig. 10 und 11, die diesem Fall entnommen sind, hinlänglich charalterisirt.

Die Gefässe des Corpus ciliare waren gut injicirt und entschieden erweitert. Der Musculus ciliar, von hypermetrop. Bau mass in seinem grössten Dickendurchmesser $0.28 \mathrm{Mm}$. (gegen $0.36 \mathrm{Mm}$, an einem gesunden 80jährigen Auge), das 
Bindegewebe zwischen den Muskelbündeln, sowie in den Processus ciliares erschien glänzend.

Der Canalis Schlemmii war auf manchen Radiärschnitten geschlossen, die Gegend desselben von Pigment infiltrirt.

Zur Beurtheilung der Blutcirculations-Verhältnisse in der Iris wurde dieselbe mit der gleichfalls von der Arteria ophthalmica aus injicirten gesunden Iris eines gleichalterigen Individunms verglichen, bei dem die Section ausgebreitetes Gefässatherom, namentlich auch im Gehirn, erwiesen hatte. Dabei ergab sich, dass auf einen gleich grossen Quadranten der gesunden Iris ungefähr 5 mal so viel injicirte radiäre Gefässstämmchen kamen als bei der glaucomatösen Iris, dass aber der Circulus irid. minor der letzteren und ebenso die erhaltenen pupillaren Capillargefässe entschieden weiter waren als in der gesunden Iris; ausserdem sah man in der Iris bei Glaucom zwischen den gefüllten Radiärgefässen eine Anzahl leere, deren Wände der hellen Farbe nach sclerotisch waren. Am linken Auge war die Injection von der Arteria ophthalmica aus nicht gelungen; als sich nun nach Enucleation beider Augen herausstellte, dass hier wahrscheinlich gleichfalls Glaucom vorliege, wurde am folgenden Tage die Injection von den Arteriae ciliares longae aus versucht*); das Auge hatte sehon 24 Stunden in Müller'scher Flüssigkeit gelegen. Es gelang nur, die Arteria ciliaris longa externa zu injiciren, die innere riss ab.

Der anatomische Befund war folgender: Vorderkammer eng. Der periphere Absehnitt der Iris liegt auf den meisten Meridionalschnitten durch den vordern Theil des Bulbus der Cornea an, ist aber nicht mit ihr verwachsen, nur die eigentliche Iriswurzel haftet etwas fester an dem abgerundeten Scheitel des Iriswinkels, in den sie hineingepresst ist

*) Da ich glaube, dass die Gefäss-Injection pathologisch veräbderter Augen für das histologische studium von Belang ist, habe ich mehrfach Injectionen mit Berliner Blau von den Arter. ciliar. longae aus versucht; denn dem Ophthalmologen wexden ja in der Regel nur enucleirte Angen zur Verfügung stehen; es gelingt in der That, wenn auch schwierig, mit sehr feinen Glascanülen, in das am normalen Auge ca. $0.04 \mathrm{Mm}$. Durchmesser betragende Lumen einzudringen und mit einer Pravaz'schen Spritze zn injiciren; leichter ist es glücklicher Weise bei Glaucom, wo die Arteriae ciliar. longae dilatirt sind (Brailey). 
(vergl. Fig. 12). Die Dicke der Iris beträgt 0.072-0.112, die radiäre Breite ca. $1.68 \mathrm{Mm}$. Das Gewebe zeigt ausgeprägte Sclerose, wenn anch nicht in der Ausdehnung wie rechts; so findet sich auch in diesem Auge der Sphincter iridis wenigstens partiell durch Sclerose des interstitiellen Bindegewebes zu Grunde gegangen; in Bezug auf Pigmentirung der Iris und Gefäss-Veränderungen verweise ich auf die allgemeine Darstellung.

Der innere Quadrant der Iris fehlt; in der Cornea findet sich daselbst eine Narbe, in die der stehen gebliebene Irisstumpf eingeheilt ist, auch in diesem Stumpf ist die Sclerose sehr deutlich und zieht sich in das etwas nach vorne gezogene Corpus ciliare hinein. Das letztere ist in der äusseren BulbusHälfte gut injicirt; die Gefässe erscheinen erweitert; die Dicke des musenl. ciliaris beträgt $0.32 \mathrm{Mm}$; das Bindegewebe zwischen den Muskelbündeln und in den Processus ciliares ist ziemlich stark glänzend.

Der Canalis Schlemmii bietet nichts Abnormes, Linse noch ziemlich durehsichtig; in der Glashaut der Chorioidea massenhafte Drusen, sonst letztere normal. Die Retina schien zur Untersuchnng nicht mehr geeignet, die Stäbchen- und Zapfen-Schicht war vollständig zerstört, wahrscheinlich cadaverös.*)

Die Sehnerven-Papille war randständig flach excavirt, der Sehnerv atrophisch bis auf einen keilförmigen Abschnitt, der in der Nähe der Papille mit seiner Basis die Peripherie erreichte und sich cerebralwärts nach dem Centralcanal des Nerven hin verzog. Das interstitielle Bindegewebe desselben zeigte sclerotische Ver"underungen.

Das Lumen der äussern langen Ciliararterie betrug im Durchmesser $0.04 \mathrm{Mm}$.

Von einer gewissen praktischen Bedeutung und deshalb erwähnenswerth scheint mir der Umstand, dass für die histologische Untersuchung der Iris für gewöhnlich eine schwache, etwa 100 fache Vergrösserung die geeignetste ist. Flächenschnitte, in dieser Weise betrachtet, geben

*) Die Leiche war schon 2 Tage alt und hatte im Hochsommer zu Section und chirurgischem Operations-Cursus gedient, ehe sie mir zur Verfügung stand. 
nicht nur ein recht prägnantes Bild der glancomatösen Veränderungen, sondern man bekommt auch den übersichtlichsten Allgemeineindruck von den histologischen Differenzen $\mathbf{z w i s c h e n ~ i n f a n t i l e r ~ u n d ~ s e n i l e r ~ I r i s . ~}$

Vielleicht lohnt es sich, hierauf noch mit zwei Worten einzugehen.

Legen wir den Flächenschnitt Fig. $10 \mathrm{zu}$ Grunde, so präsentirt sich die glaucomatöse Iris unter dem Mikroskop als ein dichtes und wegen seines (gefärbten) Zellen- und Pigment-Reichthums dunkel erscheinendes Grundgewebe, in welchem die Gefässe durch die Sclerose der zellenfreien Adventitia als weiss glänzende Bänder imponiren. In einem gewissen Gegensatz dazu steht das senile IrisGewebe: das Gefäss erscheint dunkel, weil die Wand, obgleich ebenfalls zellenarm, undurchsichtig ist, dagegen sind die Gefäss-Interstitien hell, weil sie der Hauptsache nach nur ein lockeres Netz anastomosirender Zellen entbalten: die infantile Iris endlich bietet in und um ihre Gefäss-Adventitien ein solche Fülle von Zellen, dass die Gefässe den Eindruck machen, als seien es einfach strangförmige dichtere Anhäufungen der gewöhnlichen Stromazellen der Iris; erst bei aufmerksamer Untersuchung wird in ihnen das Gefässrohr dentlich.

Die im Vorstehenden gegebene Schilderung der IrisSclerose basirt im Wesentlichen auf dem Studium von absolutem Glaucom resp. von Glaucoma inflammator. von längerer Dauer.

Wenn nun schon von ganz allgemeinem Gesichtspunkt aus anzunehmen war, dass das Anfangsstadium des Glaucoms eine gleiche Prägnanz des histologischen Bildes nicht bieten würde, so musste das für den Ophthalmologen, der weiss, wie allmälig und so zu sagen latent sich das klinische Bild des Glaucoms entwickelt, fast selbtverständlich erscheinen. 
Anderseits aber wurde schon oben darauf hingewiesen, dass es für die Bedeutung der Iris-Sclerose als ätiologisches Moment von höchster Wichtigkeit sei, den Nachweis zu erbringen, dass sie schon im früheren Stadium des Glaucoms sowie bei den einfachen Formen desselben vorhanden sei.

Um dieser Forderung zu genugen, untersuchte ich die bei Iridectomien gewonnenen Irisstuckchen in 5 Fällen von Glaucoma simplex (im weiteren Sinn) und in einem Fall von frischem Glaucoma acut. Im Allgemeinen kann ich sagen, dass alle die anatomischen Merkmale der glaucomatösen Iris, die ich oben in dem DurchsehnittsKrankheitsbild zusammengefasst habe: namentlich die Dicken-Abnahme der Iris, die Dichtigkeit und Pigmentirung des Gewebes, die Dilatation und Verschliessung der Gefässe auch hier vorhanden waren, wenn auch in geringerem Grade entwickelt. In dieser Beziehung will ich namentlich nicht verschweigen, dass die Gefässwände nicht immer die helle, glänzende Farbe zeigten, die im Verein mit dem dunkeln $Z$ wischengewebe der glaucomatösen Iris ein so in die Augen fallendes Aussehen verliehen. Indess diese Gefäss-Beschaffenheit repräsentirt offenbar zwar das Maximum der sclerotischen Wandveränderung, aber nicht das Wesen derselben. Auch in der senilen Iris zeigt ja die Gefässwand schon einen sehr ausgeprägten Untersehied gegen die infantile Iris; das Bindegewebe der Adventitia erscheint gröber, rigider, zellenarm und dürfte fast schon die Bezeichnung "senile Sclerose" rechtfertigen, obgleich die Adrentitia dabei eher dunkel erscheint.

Nach Allem, was ich gesehen, halte ich es für wahrscheinlich, dass zwischen der senilen Sclerose der Irisgefässe und zwischen der eigentlichen glaucomatösen continuirliche Uebergänge existiren und dass, wenn ein geeignetes Material, namentlich ganze Bulbi statt dürftiger Irisstückchen, eine ausgedehntere Untersuchung der Früh- 
stadien des Glaucoms ermöglichten, man ebenso ein histologisches Prodromal-Stadium constatiren würde, wie es jetzt ein klinisches giebt.

Enthält denn nun aber die Litteratur gar keine Angaben über die oben beschriebenen Irisveränderungen bei Glaucom?"

Es ist nicht zo leugnen, dass die pathologisehe Anatomie der Iris in den zablreichen anatomischen Untersuchungen über Glaucom stiefmütterlich behandelt worden ist. Das erscheint auf der einen Seite merkwürdig, weil nicht nur die ätiologisch bestgekannten Secundarglaucome an sie anknüpfen, sondern weil auch die Therapie des Glaucoms deutlich genug auf sie hinweist; auf der andern Seite wird es erklärlich, weil die normale Anatomie der Iris noch manche Lücken enthalt. Dennoch finden sich einige Notizen, wenn auch mebr beiläufig erwăhnt, nicht nur über das Gewebe der Iris im Allgemeinen, sondern auch speciell über die Gefässe.

So erwähnt H. Müller*), dass er bei Glaucom in dem peripheren, der Cornea adhaerenten Theil der Iris die Gefässe durch pigmentirte oder körnige Massen verschlossen gefunden.

E. Hache ${ }^{* *}$ ) beschreibt bei Glaucoma hämorrhagicum in dem Stroma und den Gefässwänden der Chorioidea Bindegewebswucherung, sodass die Natur der Gefässe nicht zu bestimmen war. "L'iris presente le même état. La rigidité et l'epaississement y sont plus prononcés encore, les vaisseaux offrent des altérations identiques."

Manfredi**) untersuchte einen von Gosetti wegen Glaucom enucleirten Bulbus. Er fand das Gewebe der Iris fester als normal, „die Irisgefässe waren bemerkenswerth durch den Glanz und die Homogenität ihrer Wände."

Bei Brailey t) kehrt bei Schilderung des Glaucoma

*) $1 . \mathrm{c}$.

**) Recueil d'Ophthalmol. 1875.

***) Annali di Ottalmol. 1876.

†) Ophth. Hosp. Rep. X.

v. Graefe's Archiv für Ophthalmologie, XXX. 4. 
hämorrhagicum die Bezeichnung Sclerose der Iris öfters wieder, ohne dass indess Gewicht daranf gelegt wird.

Bei früheren Autoren finden wir die Iris bisweilen als verdickt oder im Gegentheil als atrophisch bezeichnet.

Mit der Aetiologie des Glaucoms wurden, wie man sieht, die histologischen Veränderungen der Iris bis jetzt nicht in Verbindung gebracht. Dm so mehr möchte meinerseits jetzt die Frage in das Auge zu fassen sein, ob wir in denselben die Grundursache des Glaucoms zu sehen berechtigt sind. Dazu gehört der Nachweis, dass die Iris-Sclerose zeitlich vor der Drucksteigerung besteht und ferner, dass sie ihrem Wesen nach geeignet ist, die Drucksteigerung hervorzurufen.

Dass bei den Secundär-Glaucomen nach Iritis und Leucoma adhaerens die Iris der Ausgangspunkt der Drucksteigerung ist, darüber lässt schon die klinische Beobachtung keinen $\mathrm{Zweifel.} \mathrm{Obendrein} \mathrm{habe} \mathrm{ich} \mathrm{für} \mathrm{diese} \mathrm{Formen}$ anatomisch nachgewiesen, dass sich die Iris-Sclerose, mehr oder minder stark ausgebildet, schon vorfindet, ehe noch Druckerscheinungen klinisch zu erkennen sind*) und damit dürfte wohl das Causal-Verhältniss zwischen diesen beiden Factoren im Allgemeinen sicher gestellt sein.

Trotzdem habe ich versucht, die Art und Weise dieses Zusammenhangs mir auf experimentellem Wege noch klarer zu machen.

Einem albinotischen Kaninchen wurde mittelst Höllensteinstift ein grosses perforirendes Ulcus corneae beigebracht; es entstand ein typisches Leucoma adhaerens (eigentlich Melanoma adhaerens), das etwa den unteren ausseren Quadranten der Hornhaut occupirte. Das Thier blieb 18 Monate am Leben, dann wurde es Weihnachten 1883 getödtet.

Nun habe ich in der ganzen Zeit allerdings niemals Druckerscheinungen beobachtet, dagegen boten mir subcutane Fluoresein-Injectionen ein geeignetes Mittel, um den Einfluss

*) Graefe's Archiv XXVIII. 2. 
der vorderen Synechie auf die Secretion des Kammerwassers zu prüfen.

Es ergab sieh, dass sowohl im Anfang, als nachdem längst alle entzündlichen Symptome geschwunden waren, auf dem operirten Auge die Fluorescinfärbung stets früher und reichlicher in der Vorderkammer eintrat als auf dem gesunden, ja, als zuletzt, kurz vor dem Tode des Thieres, die subcutane Resorptionsfähigkeit desselben in Folge multipler subcutaner Narben wesentlich gelitten hatte, ereignete es sich einmal, dass auf dem gesunden Auge überhaupt keine Färbung zum Vorschein kam, während sie auf dem operirten Auge vollständig dentlich war; daraus darf man wohl schliessen, dass auf diesem Auge im Verbältniss zur anderen Seite eine Hypersecretion des Humor aqueus bestand.

Nach dem Tode des Thieres wurde das betreffende Auge vom Herzen aus mit einer gefärbten Leimlösung injicirt; da zeigte sich dann, dass die Processus ciliares, die der ziemlich umfangreichen Synechia anterior entsprachen, am unvollkommensten injicirt waren und dass rechts und links davon die Färbung der Processus eine mit der Entfernung wachsende Zunahme aufwies. In der dunklen Hornhaut-Iris-Narbe war von der Injectionsmasse nichts zu sehen; allerdings habe ich sie, um das Präparat zu erhalten, nicht auf Schnitten untersucht.

Wenn nun beim Secundar-Glaucom die Sclerose der Iris nach alle dem wohl mit vollem Recht als die Grundursache der Drucksteigerung angesehen werden darf, und wenn sich ferner diese Anomalie der Iris bei einer Abart desselben Leidens, beim primären Glaucom, in genau entsprechender Weise vorfindet, so hat es gewiss schon a priori viel Wahrscheinliches für sich, dass diese Veränderung hier dieselbe pathogenetische Rolle spielt wie dort, und diese Wahrscheinlichkeit wird noch erhöht dadurch, dass ich zeigte, dass schon in frühen Stadien des primären Glaucoms die Anfänge der sclerotischen Veränderungen in der Iris nachzuweisen sind.

Die Iris-Sclerose bietet aber fernerhin den Vortheil, dass aus ihr diejenigen andern pathologisch-anatomischen 
Veränderungen, die notorisch dem Glaucom zukommen und die von Einigen als Basis des glaucomatösen Processes angesprochen werden, auf das einfachste als secundärer Natur zu erklären sind: ich meine die Verlegung der Kammerbucht (Knies) und der pathologische Befund im Corpus ciliare (Brailey).

Dass die periphere vordere Iris-Synechie ein secundäres Symptom des glaucomatösen Processes sei, war von dem Augenblick an klar, wo Schnabel und Brailey nachwiesen, dass die Ursache nicht eine Entzündung des Fontana'schen Raumes wäre, sondern ein mechanisches Moment sein müsse, weil der Verwachsung eine einfache Annäherung vorausgehe. Welches dies mechanische Moment sei, dies habe ich, glaub' ich, im ersten Theil dieser Arbeit genügend erörtert, und ich verweise in dieser Beziehung auf die Beschreibung des Falles von Buphthalmus sowie von Glaucoma subacutum mit den begleitenden $\mathrm{Ab}$ bildungen; in welcher Beziehung aber die Iris-Sclerose zu diesem mechanischen Moment steht, das wird gleich unten zur Sprache kommen.

Brailey's*) Glaucom-Theorie, wie er sie am Schlusse seiner umfangreichen und eingehenden Untersuchungen aufstellt, gründet sich auf folgende T'hatsachen:

Die constantesten Veränderungen, die Brailey bei Glaucom fand und die er mit der Genese desselben in Verbindung brachte, waren die Dilatation der Gefässe, namentlich der Arterien des Corpus ciliare, die Atrophie des Ciliarmuskels und die Sclerose des zugehörigen Bindegewebes. Die Atrophie des Muskels sowie die Sclerose führt Brailey auf eine vorangegangene Entzündung zurück; allein diese Entzündung scheint mir durch seine Untersuchungen nicht erwiesen. Brailey drückt sich in dieser

*) On the nature and course of the glaucomatous Process. Ophth. Hosp. Rep. X. p. 282; vergl. auch die übrigen Arbeiten ron Brailey in demselben Band. 
Beziehung sehr vorsichtig aus. Er sagt*), dass diese Entzündung am stärksten sei in Glaucomfällen, die mit Ulcus corneae complicirt wären oder sich daran anschlössen, bei primärem Glaucom sei sie schwächer ausgebildet; niemals komme es bei derselben zu einer Verdickung des Ciliarkorpers, auch fehle jede fibrinose Exsudation; mit anderen Fntzündungsformen verglichen, zeige sie eine nur mässige Kernvermehrung in den betroffenen Theilen.

Ich kann nicht leugnen, dass nach dieser anatomischen Beschreibung die Annahme einer Cyclitis mir auf schwachen Füssen zu stehen scheint, und dieser Eindruck wird noch verstärkt, wenn wir die klinische Beobachtung zu Rathe ziehen. Nach Brailey müsste die Cyclitis in das Prodromalstadium des Glaucoms fallen, allein mir scheint es doch, als seien die Symptome dieser sich bisweilen uber Jahre hinziehenden Periode andere, als wir sie yon einer selbst schleichenden Cyclitis zu sehen gewöhnt sind.

Aber ganz abgeseben davon, bleibt Brailey die Erklärung für die Gefässdilatation im Corpus ciliare schuldig. In dieser Beziehung sind seine Aeusserungen etwas unbestimmter Natur. Nachdem er in einer frühern Arbeit**) eine Gefäss-Neurose als møgliche Ursache davon hingestellt hatte, drückt er sich später mehr negativ aus. Er bemerkt, dass die Gefäss-Dilatation zu der Entzündung nicht in directem Abhängigkeits-Verhältniss stehe; dafür sei die Wandverdünnung der Gefässe zu ausgesprochen; man könne aber auch nicht annehmen, dass sie die Folge der Tensions-Zunahme des Bulbus sei; denn sie sei oft beschränkt auf einen Theil des Corpus ciliare, während der andere frei bleibe. $* * *$ )

Alle diese Schwierigkeiten fallen nun weg, sobald man die Sclerose der Iris als das primäre Glied in der

*) 1. c. p. 90 .

**) I. c. p. 12.

***) 1. c. p. 91 . 
Kette der Glaucom-Erscheinungen annimmt; der gewőhnliche anatomische Befund bei Glaucom erklärt sich alsdann auf das einfachste.

Die Selerose der Iris bewirkt auf der einen Seite durch Betheiligung des Gefäss-Systems Circulationsstörungen in der Iris. Hier kommt es zu Verschluss von Gefăssen durch Compression oder Thrombose, dort entwickeln sich compensatorische Dilatationen. Die Circulation wird erschwert; namentlich in den pupillaren Endschlingen entstehen chronische Blutstauungen und diese führen zu der allgemeinen Pigmentirung des Irisgewebes.

Diese Circulationsstörung beschränkt sich aber nicht auf die Iris, sondern sie übt einen rückwirkenden Einfluss auch auf das Quellgebiet im Corpus ciliare; sie führt hier zu Erweiterung der Arterien, für die Brailey als Maasstab die Dilatation des Circul. irid. major fast constant nachgewiesen hat. Auf diese chronische Blutüberfüllung ist die schliesslich eintretende Atrophie des Ciliarmuskels sowie die Sclerose des Bindegewebes ungezwungen zurückzuführen, ohne dass man eine besondere Cyclitis zu supponiren braucht.

Indess noch weiter zurück macht sich die Circulationsstorung in der Iris in der Erweiterung der Arter. eiliar. long. und in den entsprechend verlaufenden Seleral-Staphylomen bemerklich, und ich darf bei dieser Gelegenheit wohl daran erinnern, dass ich schon in meiner ersten Arbeit*) hervorgehoben, wie bei subeutanen :Injectionen von Ferro-Cyan-Kalium sich in auffallender Weise längs der Arter. ciliar. longae des Kaninchenauges ein blauer Streifen hinzog als Zeichen lebhafter Exsudation.

Die Sclerose der Iris führt aber andererseits zu Verdichtung des Gewebes und beeinträchtigt seine FiltrationsFähigkeit. Als Ausdruck der erschwerten Iris-Durch-

*) 1. c. Ueber die Ernährung des Auges p. 38. 
querung seitens des in vermehrter Menge secernirten Kammerwassers sehen wir zunächst die Annäherung der Irisperipherie an die Cornea, später die Verwachsung zu Stande kommen. Nur so ist die auffallende Faserrichtung und die merkwürdige Formverănderung zu erklären, die schon Schnabel an der Iriswurzel erwähnt und von der ich ein so uberzengendes Beispiel in dem Falle von Buphthalmus anführen konnte. Im Weiter-Verlauf kommt es dann zu Verdichtung und Atrophie der Iriswurzel.

Die übrigen Factoren des anatomisch-pathologischen Befundes bei Glaucom sind in bekannter Weise entweder auf die Drucksteigerung oder auf Complicationen zurückzuführen, so namentlich die Excavation der Papille und die Staphylome (wenigstens der Hauptsache nach) und andererseits die Netzhautblutungen etc. (Sclerose der Retinalgefässe).

Ein unbefangenes Urtheil wird sich dem Eindruck nicht verschliessen können, dass von den einzelnen Factoren, welche das complexe anatomische Bild des Glaucoms zusammensetzen, sobald man von der Iris-Selerose als Ausgangspunkt ausgeht, sich zwanglos einer dem andern anfügt.

Auf diesen Ausgangspunkt weist uns endlich auch die kliniscbe Beobachtung hin.

Während Brailey eine Cyclitis zu Hülfe nehmen muss, um die Druckzunahme im Prodromalstadium zu erklären, eine Cyelitis, von der der Kliniker nichts weiss und die übrigens nach ihrem Uebergang in Sclerose die Rolle der Druckvermebrung wieder abgiebt und die Annahme eines andern vicariirenden Factors nothig macht, so findet bei Zugrundelegung der Iris-Sclerose als primaren Factors gerade die Eigenthümlichkeit des Prodromalstadiums die ungezwungenste Erklärung. Wenn uns die Praxis hundertfaltig zeigt, wie an einem scheinbar gesunden 
Auge nach künstlicher Erweiterung der Pupille plötzlich Glaucom ausbricht und wie anderseits der Prodromalanfall durch einen Tropfen Eserin coupirt wird, so wird man doch geradezu auf die Iris als auslosendes Moment des Glaucom hingestossen.

Die ungezwungene Natürlichkeit, mit welcher bei Annahme der Iris-Sclerose nicht nur dieser Umstand, sondern fast alle selbst unwesentlichen Punkte der Phänomenologie des Glaucoms ihre Deutung finden, scheint mir als Stütze für diese Glaucomtheorie überhaupt nicht zu unterschătzen. Es genügt nicht, dass eine Theorie sich allein auf den Sectionsbefund stutzt, es muss dieselbe auch zu dem ganzen Verlauf der Krankheit von Anfang bis zu Ende, es muss dieselbe zu denjenigen Krankheitserscheinungen, die ihrer Natur nach in dem Sectionsbefund nicht zum Ausdruck kommen, es muss dieselbe endlich zu den Erfolgen unserer Therapie in Uebereinstimmung gebracht werden können. Diese Forderung ist gerechtfertigt, weil die klinische Seite der Glaucomlehre seit lange so genau gekannt, so weit der pathologischen Anatomie vorausgeeilt ist, dass sie sich vorzugsweise als Prufstein eignet für die Richtigkeit einer Theorie, die wesentlich auf Grund anatomischer und experimenteller Studien aufgebaut ist.

Von diesem Standpunkt aus habe ich schon früher*) die Irisfiltration einer eingehenden Betrachtung unterzogen, und es soll nur zur Ergänzung dieser Betrachtung dienen, wenn ich im Nachstehenden prüfe, ob die Annahme der Iris-Sclerose mit der klinischen Erscheinungsweise des Glaucoms in demselben Maasse stimmt wie mit dem pathologisch-anatomischen Befund.

Das Glaucom ist im Allgemeinen eine Krankheit der reifern Jahre; wir wissen, dass auch vordere und hintere

*) 1. c. Ueber die Ernährung des Auges. 
Synechieen es lieben, erst im höhern Alter zu Secundärglancom zu führen. Wir finden ferner das Glaucom meist doppelseitig und bisweilen erblich.

Es genügt, diesen Eigenthümlichkeiten gegenüber darauf hinzuweisen, dass die Iris-Selerose in ihrer schwächsten Form seniler Natur ist und dass Doppelseitigkeit und Heredität hier ebensowenig Ueberraschendes haben als bei Gefäss-Atherom und Gehirn-Apoplexie.

Unter den Refractionen zeichnet sich die Myopie durch eine gewisse Immunităt aus. Hier lässt meiner Ansicht nach die Irissclerose im Stich, ein neues Moment muss zur Erklärung herangezogen werden, die Festigkeit der Sclera.

Ich kann auf Grund eigener Messungen Brailey nur beistimmen, dass die Selera bei Glancom nicht rigider erscheint als bei anderen emmetropischen und hyperopischen Augen älterer Individuen; aber meiner Auffassung nach handelt es sich auch gar nicht darum, an der Sclera glaucomatös erkrankter emmetropischer und hyperopischer Augen eine besondere Praedisposition zu entdecken, sondern vielmehr bei den Sclerae myopischer Augen nach dem Grund der relativen Immunität zu forschen.

Ich hatte Gelegenheit, in dieser Beziehung das myopische Auge eines 70jährigen Individuums mit den mir zur Disposition stehenden Glaucom-Augen zu vergleichen. Es wurden Dickenmessungen der Sclera ausgeführt, und zwar so, dass meridionale Streifen derselben, die entsprechenden Bulbus-Meridianen der verschiedenen Augen entnommen waren, auf einander gepresst und in Querschnitte zerlegt wurden. Dabei ergab sich, dass die Sclera des myopischen Auges deutlich dünner war als die der Glaucom-Augen, und es scheint demnach nicht unwahrscheinlich, dass diese Dünnheit und Dehnbarkeit der Sclera, wie sie der Grund der erworbenen Myopie ist, 
anderseits bis zu einem gewissen Grad das Zustandekommen von pathologischer Drucksteigerung verhütet.

Das Glaucom entwickelt sich im Allgemeinen langsam und allmälig. Trăge Pupillen-Reaction, Einschränkung der Accomodationsbreite gebören zu den frühesten praemonitorischen Symptomen, die sich namentlich während der Glaucom-Anfälle steigern. Die Veranlassungen zu letzteren sind entweder der Art, dass sie zu Pupillenerweiterung führen oder den Blutdruck herabsetzen (Herzschwache: Weber, Landsberg).

Da die Circulationsstörungen, welche die Irissclerose mit sich führt, sich namentlich im Sphincter iridis und im Musculus ciliaris abspielen, da, wie ich oben hervorbob, in der Nähe des bei Glaucom erweiterten Circul. arter. irid. major zahlreiche Irisnerven vorbeipassiren, so ist es nicht schwer zu verstehen, wie die Function der genannten Muskeln leiden kann. Sinkt der Blutdruck bei Krankheiten und dergl., so nimmt die Circulationsstörung in Iris und Corpus ciliare zu, am mächtigsten aber muss in dieser Beziehung Pupillendilatation einwirken.

Als hervorstechendes Merkmal der Mydriasis zeigt die anatomische Untersuchung atropinisirter Augen eine enorme Schlängelung der radiären Irisgefässe und eine Dickenzunahme der Membran. Nun, man braucht sich blos zu erinnern, dass die Chirurgen starke Flexionsstellung des Extremitäten zur Blutstillung empfehlen, um zu begreifen, welche Einwirkung die starke Schlängelung der Irisgefăsse auf die Cireulationsverhältnisse ausüben wird.

Auf die übrigen Symptome des Glaucoms gehe ich hier nicht näher ein. $\mathrm{Ob}$ bei dem Zustandekommen der Sehnerven-Excavation ausser dem intraocularen Druck auch die Entzündung und Sclerose des Bindegewebes des Centralcanals und der Septen eine Rolle spielt (Brailey) und ob sich hierbei vielleicht die früher erwähnten Resultate der intraocularen Tusche-Injectionen, die Verschleppung 
der Farbstofftheilchen in den Sehnerven, verwenden lassen, bleibe dahingestellt.

Auch auf die Wirkungsweise der Iridectomie und der Pupillen-verengernden Mittel sei mit Rücksicht auf die frühere Besprechung nur kurz hingewiesen. Bei Verengerung der Pupille werden die Irisgefässe gestreckt, die Iris verdünnt und angespannt; so wird die Circulation des Blutes erleichtert, die Kammerwasser-Filtration begünstigt.

Zum Schlusse möge es mir gestattet sein, meine Glaucom-Theorie in einige kurze Satze zusammenzufassen:

Das Glaucom beruht auf Bindegewebs-Sclerose der Iris. Dieselbe entwickelt sich entweder im Anschluss an entzündliche Erkrankungen der Iris oder primär im höhern Alter, indem die gewöhnliche Altersveränderung der Iris, wie es scheint, bei besonderer Prädisposition allmälig einen pathologischen Charakter annimmt. Durch Betheiligung des Gefäss-Systems an der Sclerose entstehen Circulationsstörungen in der Iris und diese pflanzen sich ruckwärts auf das Corpus ciliare fort. Es entwickelt sich hier chronische Hyperämie mit Gefäss-Dilatation; der Muscul. ciliar. wird schliesslich atrophisch, das Bindegewebe sclerotisch.

Die Folge der Hyperämie ist Hypersecretion des Humor aqueus und Drucksteigerung. Linse und Iris werden in Folge dessen nach vorne und fest aufeinander gepresst. Wenn hierdurch der Abfluss des Kammerwassers durch die Pupille verhindert wird, so führt anderseits die Iris-Sclerose durch Verdichtung des Gewebes zu verminderter Filtrationsfahigkeit: die periphere Iris-Durchquerung seitens des Humor aqueus leidet. Der perilentale Flüssigkeitsstrom drängt die Iriswurzel an die Cornea; die Kammerbucht wird verschlossen und damit der Abfluss des Kammerwassers geschädigt: die Druckzunahme wächst. Wird zeitweilig durch Mydriasis oder Herzschwäche die Circulationsstörung in Iris und Corpus ciliare vermehrt, so ent- 
Dr. Rich. Ulrich.

steht eine acute Steigerung aller Symptome, ein GlaucomAnfall, und umgekehrt ist derselbe durch myotische Mittel momentan zu beseitigen, indem die Streckung der Irisgefässe die Circulation erleichtert. Die Heilwirkung der Iridectomie beruht auf der partiellen Entfernung der sclerotischen Iris, d. h. auf der Wegräumung des Hemmnisses, welches sich dem perilentalen Flüssigkeitsstrom entgegenstellt. 


\section{Anhang.}

Wie man erkennt, beruht nach dieser Theorie der glaucomatose Process im Wesentlichen auf einer Vermehrung der Secretion, die von der Iris aus gleichsam ausgelöst wird. Nun wäre es eigenthümlich, wenn eine solche Hypersecretion nicht auch gelegentlich auf anderem Wege zu Stande kommen sollte.

In dex That wirkt gewiss schon bei Leucoma adhaerens die Zerrung des Corpus ciliare irritirend und hyperämisirend, d. h. mithelfend in Bezug auf die Genese der Drucksteigerung; die Chorioiditis externa hämorrhagica, die ich oben in dem Falle von Buphthalmus beschrieben, muss gewiss als geeignet anerkannt werden, Drucksteigerung zu erzeugen, und in analoger Weise ist die Wirkungsart intraocularer Tumoren und dislocirter Linsen aufzufassen. Des Beispiels halber mag ein Fall von Glaucom nach traumatischer Luxation der Linse, den ich anatomisch untersuchen konnte, hier angeführt werden.

Die klinische Diagnose lautete:

Iridochorioiditis traumat., Narbe der Cornea, tiefe Vorderkammer, Iridodonesis, $\mathrm{T}+\mathbf{1}$.

Bei der anatomischen Untersuchung (Fig. 13) fand sich makroskopisch die Iris mit der vorderen Linsenkapsel verwachsen; die hintere Linsenkapsel lag der vorderen z. Th. dicht an, so dass also sehr wenig eigentliche Linsenmasse vorhanden war. Dagegen fand sich nach Wegräumung des gelatinösen Glaskörpers in der Năhe der Ora serrata ein linsenähnlicher Körper, der Retina anliegend. Die Processus ciliares dieser Gegend erschienen rostbraun, der Glaskörper haftete in seinem vorderen Theil besonders fest an der Retina.

Mikroskopisch fand sich an der mir zur Verfügung stehenden Bulbushälfte die Cornea normal, dagegen starke 
pericorneale Hyperämie und Zelleninfiltration der Conjunctiva. Die Iris in normaler Lage, die Kammerbucht, die Fontana'schen Räume und der Schlemm'sche Canal von zellenreichem Exsudat occupirt. Die Iris sehr hyperämisch, sehr zellenreich, besonders im peripheren Theil, dieser auch, anscheinend rom Urealblatt aus, von Pigment invahirt. Die hintere Kammer angefüllt von $z$. Th. eitrigem Exsudat, namentlich zeigen die Processus ciliares und das Corpus ciliare einen eitrigen Belag; die Fasern der Zonula sind zellig infiltrirt.

Zwischen der vordern und hintern Linsenkapsel ein eigenthümlicher Tnhalt von grossen mattglänzenden Kugeln und unregelmässig zerstreuten Zellen, nur an der Innenseite der Vorderkapsel, der das Epithel fehlt, Reste von gequollenen Linsenfasern. Die hintere Linsenkapsel zeigt eine Ruptur, die Rissränder sind nach hinten aufgerollt.

Die Hyperämie des Corpus ciliare und des vorderen Theils der Chorioidea war am stärksten in der Nachbarschaft der dislocirten Linse, fand sich aber, wenn auch abnehmend, in dem ganzen entsprechenden Aequatorialkreis. Als Grund der rostbraunen Farbe der Process. ciliares ergab sich eine Pigmentinfiltration von den Zellen des Stratum pigmentos. aus in die gewucherten Zellen der Pars ciliar. retinae resp. weiter hinten in die Stützfasern, die mikroskopisch goldgelb aussahen.

Diese Pigmentinfiltration war am deutlichsten da, wo die Linse der Retina auflag; hier waren die eigentlichen Pigmentzellen vollständig entfärbt und dafür die Stützfasern pigmentinfiltrirt, so dass dadurch eine gewisse regelmässige, pallisadenartige Anordnung des verschleppten Pigments zu Stande kam.

Hier war auch Retina und Chorioidea am stärksten verändert, beide Membranen durch Hyperämie und Zellen-Anhäufungen, die Retina ausserdem noch durch Wucherung der radiären Fasern ausserordentlich verdickt, die letztere zum Theil durch ein Exsudat von der Chorioidea abgehoben.

Die Linse selbst ist von einem Exsudat umgeben, das Zellen der mannigfaltigsten Form enthält; dieselben haben die Oberfläche der Linse usurirt; letztere selbst ist getrübt, die Linsenfaserung kaum zu erkennen. Der vordere Theil des Corpus vitreum ist von Exsudat durchsetzt.

Diese entzündlichen Veränderungen nehmen nach dem Aequator zu rasch ab. In der Chorioidea treten hin und wieder noch einzelne Lymphzellen-Anhäufungen auf. Die Retina 
nimmt ihre normale Dicke und Schichtung wieder an, bleibt aber weithin von Pigment infiltrirt, das, offenbar von dem Entzündungsherd stammend, zunächst diesem ganz diffus vertheilt ist, sich aber weiter nach hinten allmälig auf die Umgebung der Gefässwände concentrirt. Die Stäbchen und Zapfen aber sind weithin, zum Theil noch am hinteren Augenpol, vollständig zerstört.

In diesem Falle liegt die Pathogenese der glaucomatösen Drucksteigerung auf der Hand; eine regionäre Chorioiditis exsudativa verursacht eine entzündliche Hypersecretion, die das Pigment des Stratum pigmentos. in die Retina verschleppt und den ganzen vordern Bulbusabschnitt mit Exsudat anfullt.

Solchergestalt mag wohl auch die Entstehung des Glaucoms bei intra-ocularen Tumoren sein.

Im September 1884. 


\section{Erklärung der Abbildungen.}

Fig. 1. Kaninchenauge, vorderer Abschnitt. T Corpus eiliare von Tusche infiltrirt. S Vordere Synechie.

Fig. 2. Linse und Gla-körper desselben Auges. T Tusche.

Fig. 3. Meridionalschnitt*) durch die Gegend des Iriswinkels. T Tusche.

Fig. 4. Meridionalschnitt durch den vordern Theil des Auges bei Buphthalmus. C Cornea. I Iris.

Fig. 5. Irisgefäss, senil, Querschnitt.

Fig. 6. Grosse Iris-Arterie.

Fig. 7. Kindliche Iris-Arterie, Querschnitt.

Fig. 8. Marklose Nervenfaser mit Bindegewebsscheide. Querschnitt.

Fig. 9. Dasselbe. Flächenschnitt der Iris.

Fig. 10. Flächenschnitt der Iris. $\nabla$ Gefäss mit sclerotischer Wandung. Glancoma absol.

Fig. 11. Pigmentthrombus in einem selerotischen Gefüss. Glauc. absolutum.

Fig. 12. Glaucoma subacutum. Iriswurzel in die Kammerbucht gedrängt. Gefässe injicirt.

Fig. 13. Linsenluxation in den Glaskörper. Ch Chorioidea. R Retina. I Linse.

*) Der Schnitt ist nicht ganz streng meridional, da er aus der Mitte einer Schnittserie stammt. 

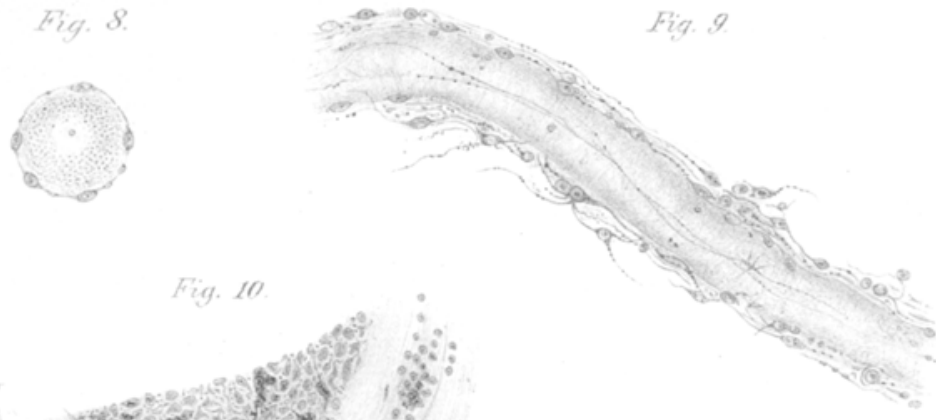

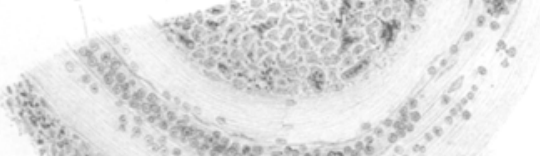

\section{Fig. 10}
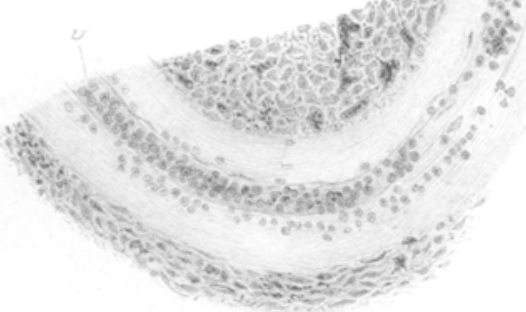

$$
\text { Fig. } 11
$$
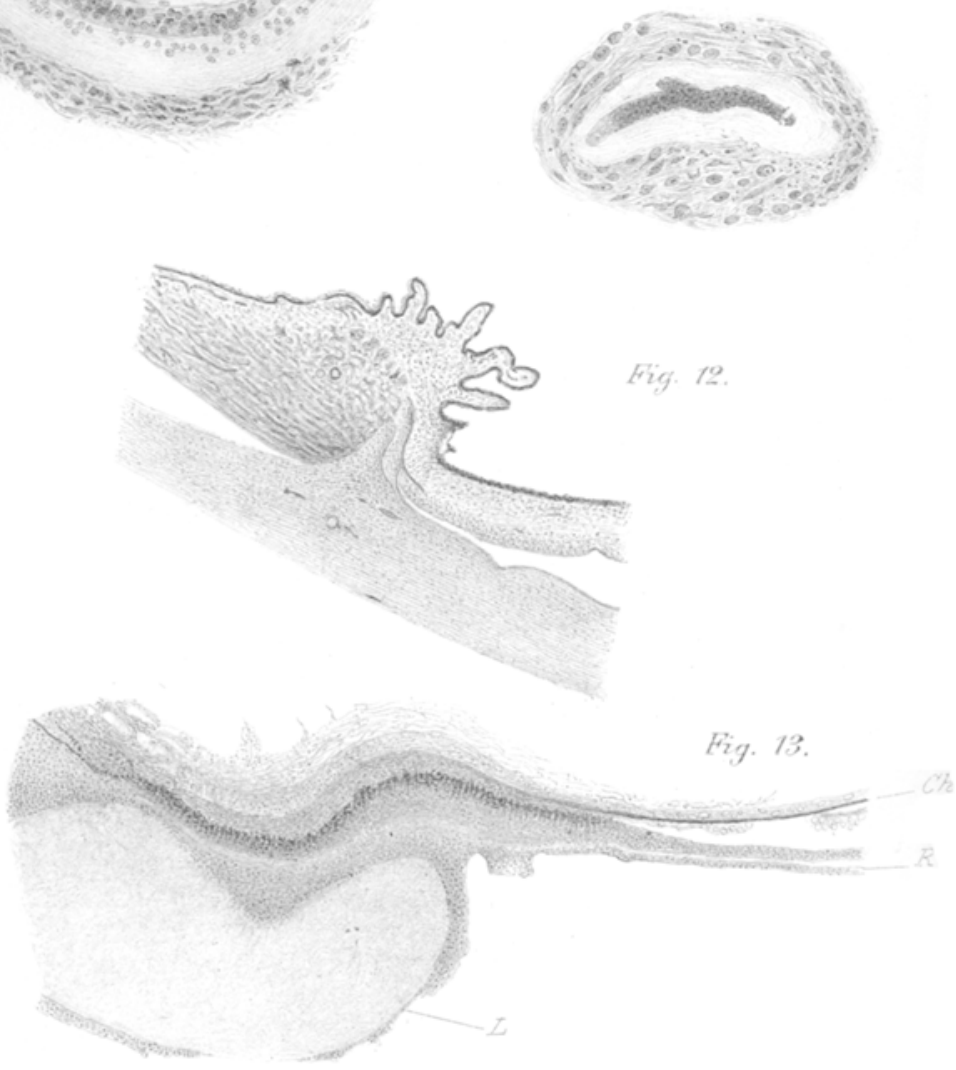

Fig. 12.

Fig. 13. 

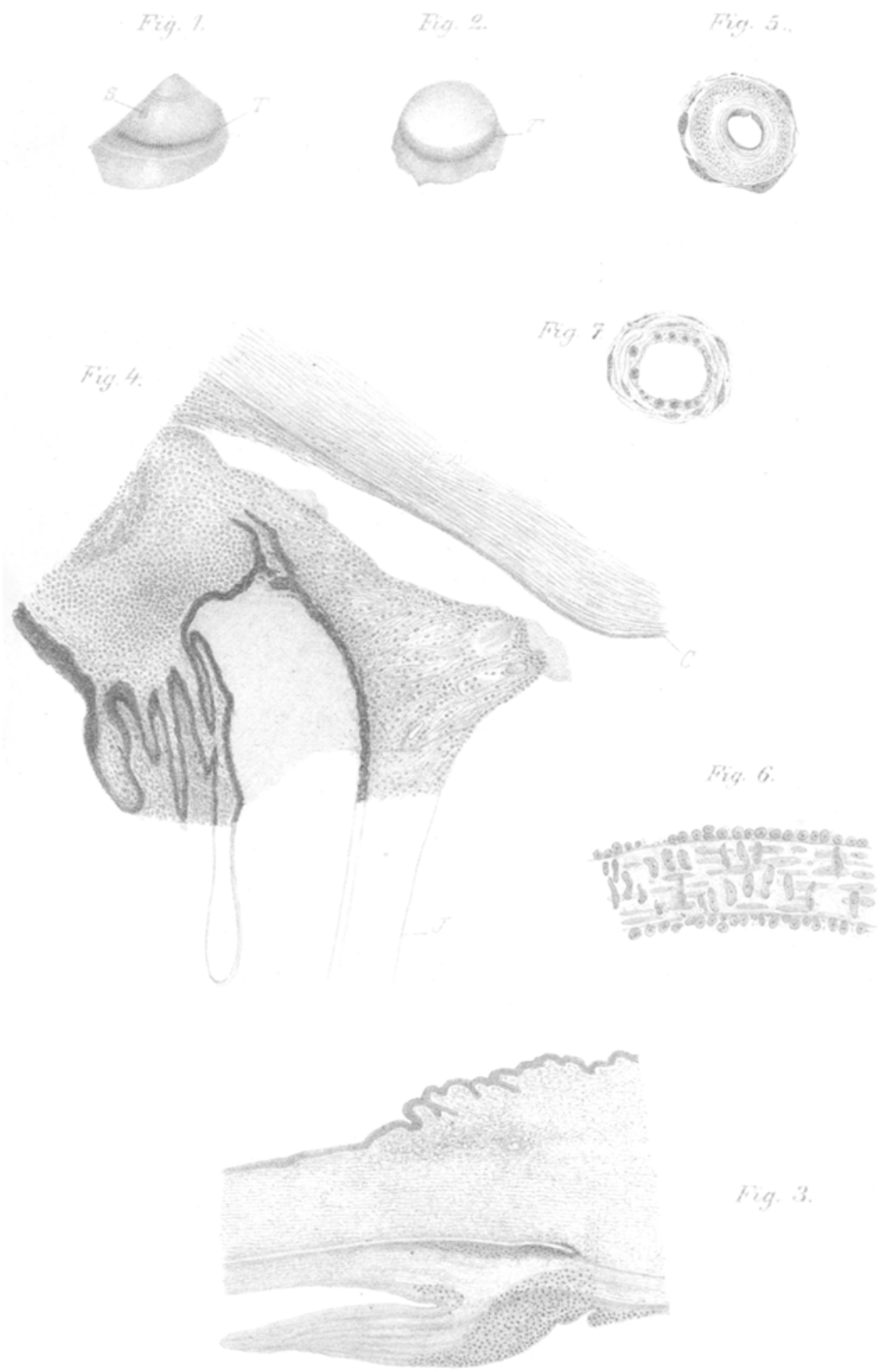